\title{
Genetic Dissection of the RNA Polymerase II Transcription Cycle
}

\author{
Shao-Pei Chou ${ }^{1}$, Adriana K. Alexander ${ }^{1,2}$, Edward J. Rice ${ }^{1}$, Lauren A Choate ${ }^{1}$, Paula E Cohen ${ }^{2}$, \\ and Charles G. Danko ${ }^{1,2, *}$ \\ ${ }^{1}$ Baker Institute for Animal Health, College of Veterinary Medicine, Cornell University, Ithaca, NY 14853. \\ ${ }^{2}$ Department of Biomedical Sciences, College of Veterinary Medicine, Cornell University, Ithaca, NY 14853.
}

\section{*Address correspondence to:}

Charles G. Danko, Ph.D.

Baker Institute for Animal Health

Cornell University

Hungerford Hill Rd.

Ithaca, NY 14853

Phone: (607) 256-5620

E-mail: dankoc@gmail.com

\section{Abstract}

How DNA sequence affects the dynamics and position of RNA Polymerase II during transcription remains poorly understood. Here we used naturally occurring genetic variation in F1 hybrid mice to explore how DNA sequence differences affect the genome-wide distribution of Pol II. We measured the position and orientation of Pol II in eight organs collected from heterozygous F1 hybrid mice using ChRO-seq. Our data revealed a strong genetic basis for the precise coordinates of transcription initiation and promoter proximal pause, which was composed of both existing and novel DNA sequence motifs, and allowed us to redefine molecular models of core transcriptional processes. Our results implicate the strength of base pairing between A-T or G-C dinucleotides as key determinants to the position of Pol II initiation and pause. We reveal substantial differences in the position of transcription termination, which frequently do not affect the composition of the mature mRNA. Finally, we identified frequent, organ-specific changes in transcription that affect mRNA and ncRNA expression across broad genomic domains. Collectively, we reveal how DNA sequences shape core transcriptional processes at single nucleotide resolution in mammals. 


\section{Introduction}

Transcription by RNA polymerase II (Pol II) is the core process responsible for producing mRNA for all protein-coding genes and most non-coding RNAs (ncRNAs). Transcription by Pol II is a highly stereotyped and cyclic process (Fuda et al., 2009; Jonkers and Lis, 2015). During the Pol II transcription cycle, RNA polymerase is initiated on regions of accessible chromatin by the collective actions of transcription factors and co-factors that recruit the pre-initiation complex, melt DNA, and initiate Pol II (Grünberg et al., 2012; Haberle and Stark, 2018; Murakami et al., 2013; Tsai and Sigler, 2000). After initiation, Pol II pauses near the transcription start site of all genes in most metazoan genomes (Jonkers et al., 2014; Muse et al., 2007; Rougvie and Lis, 1988). Pol II is released from pause through the collective actions of transcription factors and a key protein kinase complex (P-TEFb), a tightly regulated step that controls the rates of mRNA production (Danko et al., 2013; Dig B. Mahat et al., 2016; Rahl et al., 2010; Zeitlinger et al., 2007). After pause release, Pol II elongates through gene bodies, which in some cases cover more than $1 \mathrm{MB}$ of DNA in mammals (Carninci et al., 2005). Finally the co-transcriptionally processed pre-mRNA is cleaved from the elongating Pol II complex, allowing termination and recycling of Pol II (Cho et al., 1999; O'Sullivan et al., 2004; Rosonina et al., 2006).

Each of these core stages of Pol II transcription require the coordinated efforts of dozens of macromolecules. Through outstanding research achievements during the past decades, we have a new appreciation of the molecular players involved in each stage of the transcription cycle (Gilchrist et al., 2010; Miller et al., 2001; Nechaev et al., 2010; Orphanides et al., 1998; Ranish et al., 1999), as well as the dynamics of individual stages (Danko et al., 2013; Gressel et al., 2019, 2017; Jonkers et al., 2014; Schwalb et al., 2016).

Despite an expanding knowledge of the macromolecules involved in each stage of transcription, we still have a relatively rudimentary understanding about how DNA sequences influence each step in the Pol II transcription cycle. Previous studies have identified core promoter motifs that are correlated with transcription initiation, such as the TATA box and the initiator motif (Carninci et al., 2006; Smale and Baltimore, 1989). Likewise, weak motifs that correlate with pausing and Pol II termination are reported (Gressel et al., 2017; Schwalb et al., 2016; Tome et al., 2018). These DNA sequences that underlie initiation play an important role in gene regulation, and SNPs affecting these core transcriptional processes can alter the rates of mRNA production (Kristjánsdóttir et al., 2020). However, the DNA sequence specificity of the core promoter is extremely weak, degenerate, and spread across the promoter region. As a consequence, we are currently unable to predict how specific DNA sequence changes will affect the core transcriptional processes involved in transcription.

Here we use naturally occurring genetic variation between highly heterozygous $\mathrm{F} 1$ hybrid mice to understand how DNA sequence differences between alleles affect the Pol II transcription cycle. We generated an atlas of the position and orientation of RNA polymerase II in eight organs collected from three primary germ layers, using $\mathrm{ChRO}$-seq to identify the position and orientation of RNA polymerase (Chu et al., 2018). Our results provide insight into how DNA sequences shape transcription initiation and pausing. We reveal substantial, heritable differences in the position of transcription termination, which frequently do not affect the composition of the mature mRNA. Finally, we identified frequent, organ-specific changes in transcription that affect mRNA and ncRNA expression across broad genomic domains. Collectively, our results provide new insight into how genetics shape core transcriptional processes in mammals. 


\section{Results}

\section{Atlas of allele specific transcription in F1 hybrid murine organs}

We obtained reciprocal F1 hybrids from two heterozygous mouse strains, C57BL/6 (B6) and Castaneus (CAST) (Fig. 1A). Mice were harvested in the morning of postnatal day 22 to 25 from seven independent crosses (3x C57BL/6 x CAST and 4x CAST x C57BL/6; all males). We measured the position and orientation of RNA polymerase genome-wide in 8 organs using a $\mathrm{ChRO}$-seq protocol that provides accurate allelic mapping by extending the length of reads using strategies similar to length extension ChRO-seq (Chu et al., 2018) (see Methods). We obtained 376 million uniquely mapped ChRO-seq reads across all eight organs (21-86 million reads per organ; Supplementary Table 1) after sequencing, filtering, and mapping short reads to individual B6 and CAST genomes (see Methods). Hierarchical clustering using Spearman's rank correlation of ChRO-seq reads in GENCODE annotated gene bodies (v.M25) grouped samples from the same organ. Additionally, organs with similarities in organ function clustered together, for instance: heart and skeletal muscle, and large intestine and stomach (Fig. 1B).

Using ChRO-seq data from all eight organs, we identified 3,494 broad domains that showed consistent evidence of allelic imbalance across biological replicates (Fig. 1C, Supplementary Table 2). Using the reciprocal hybrid cross design, we found that the majority of these domains ( $n=3,466$; Fig. 1D) have consistent effects in each mouse strain (called straineffect domains), the pattern expected if allelic imbalance was caused by DNA sequence differences between strains. Twenty-eight domains showed consistent evidence of genomic imprinting (imprinted domains). Both strain-effect and imprinted domains were generally composed of multiple transcription units, including annotated genes (Fig. 1E) and ncRNAs (either long intergenic ncRNAs and/ or enhancer-templated RNAs). For instance, the imprinted domain associated with Angelman syndrome consisted of twenty ncRNAs and four genes that were consistently transcribed more highly from the paternal allele and two genes transcribed from the maternal allele in brain tissue (Fig. 1F). On average, both strain-effect and imprinted domains spanned broad genomic regions ( 10-1,000 kb; Fig. 1G) which were reminiscent of regional, coordinated effects across functional elements (Delaneau et al., 2019; Rennie et al., 2018).

Recent reports in humans suggest that organs frequently share common genetic factors that shape gene expression (GTEx Consortium et al., 2017). In contrast to these recent observations, nearly $70 \%$ of strain-effect domains and $50 \%$ of imprinted domains were organ specific (Fig. 1H). Organ-specific allelic biased domains were not dominated by false-negatives in putatively unbiased organs, as the magnitude of allelic balance in putatively unbiased organs was distributed around 0 (Supplementary Figure 1A,B). Organ-specific allelic bias also could not be explained by organ-specific differences in gene expression, as organs that showed no evidence of allelic bias frequently had similar or higher transcription levels of allelic biased genes (Supplementary Figure 1C). Thus, allelic bias is organized in large multi-transcript domains that are often organ-specific.

\section{Enhanced genomic imprinting in murine brain}

Our reciprocal cross design allowed us to define 28 domains that show clear evidence of genomic imprinting, most of which were previously reported by others (Andergassen et al., 2017; 
BbioRxikprequint dodistas://dgigrg/10.1101/2021.05.23.445279; this version posted May 30, 2021. The copyright holder has placed this

A B Beprint (Wheth was notengified Bypeer revew) in the Public Domain. It is no longer restricted by copyright. Anyone can legally share, reuse,

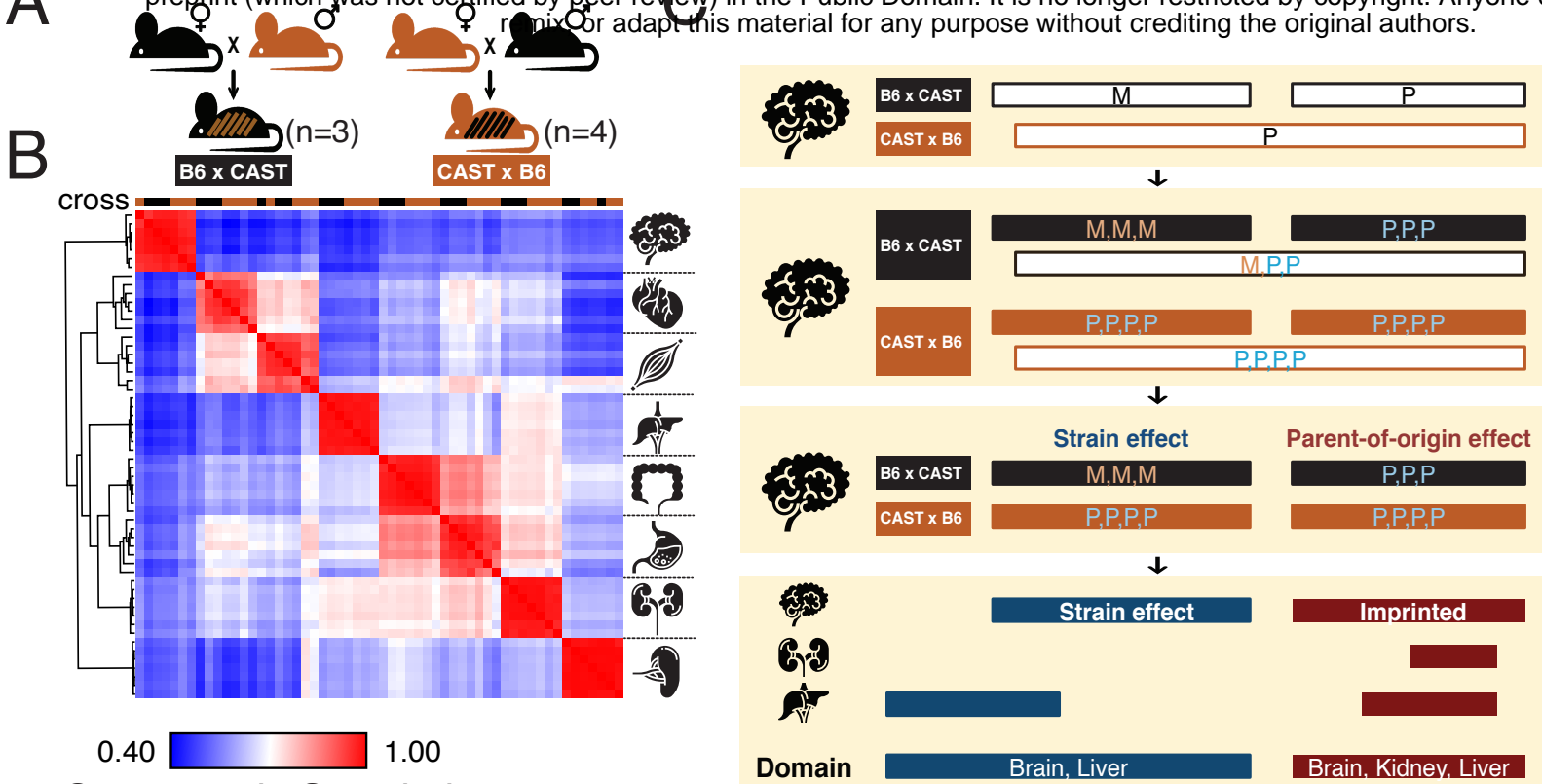

Spearman's Correlation

$\mathrm{D}$

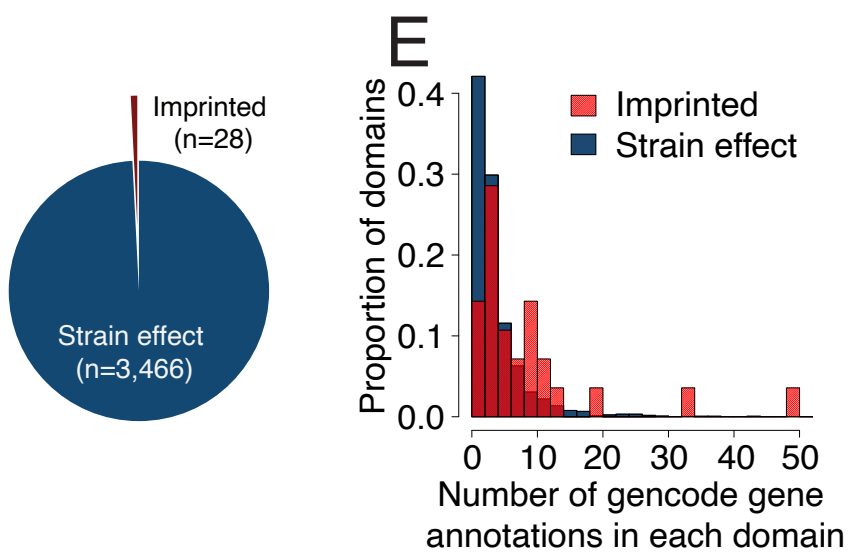

Identify candidate AlleleHMM blocks using pooled ChRO-seq

Combined blocks above from the same organ and examine the allelic bias with biological replicates. Keep the blocks that are consistently biased within both cross.

Determine whether the blocks are under strain effect or parent-of-origin (imprinted) effect.

Merged overlapped blocks from different organs into domains.

\section{Imprinted domain}

Imprinted protein-coding gene
G

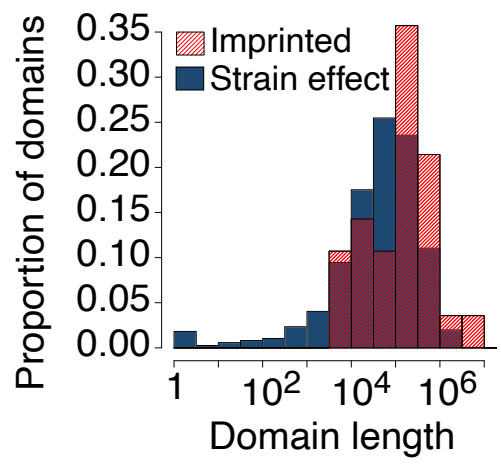

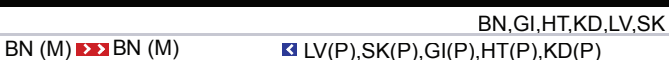

$$
\text { BN }(\mathrm{P}) \quad \mathrm{BN}(\mathrm{P})
$$

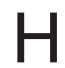

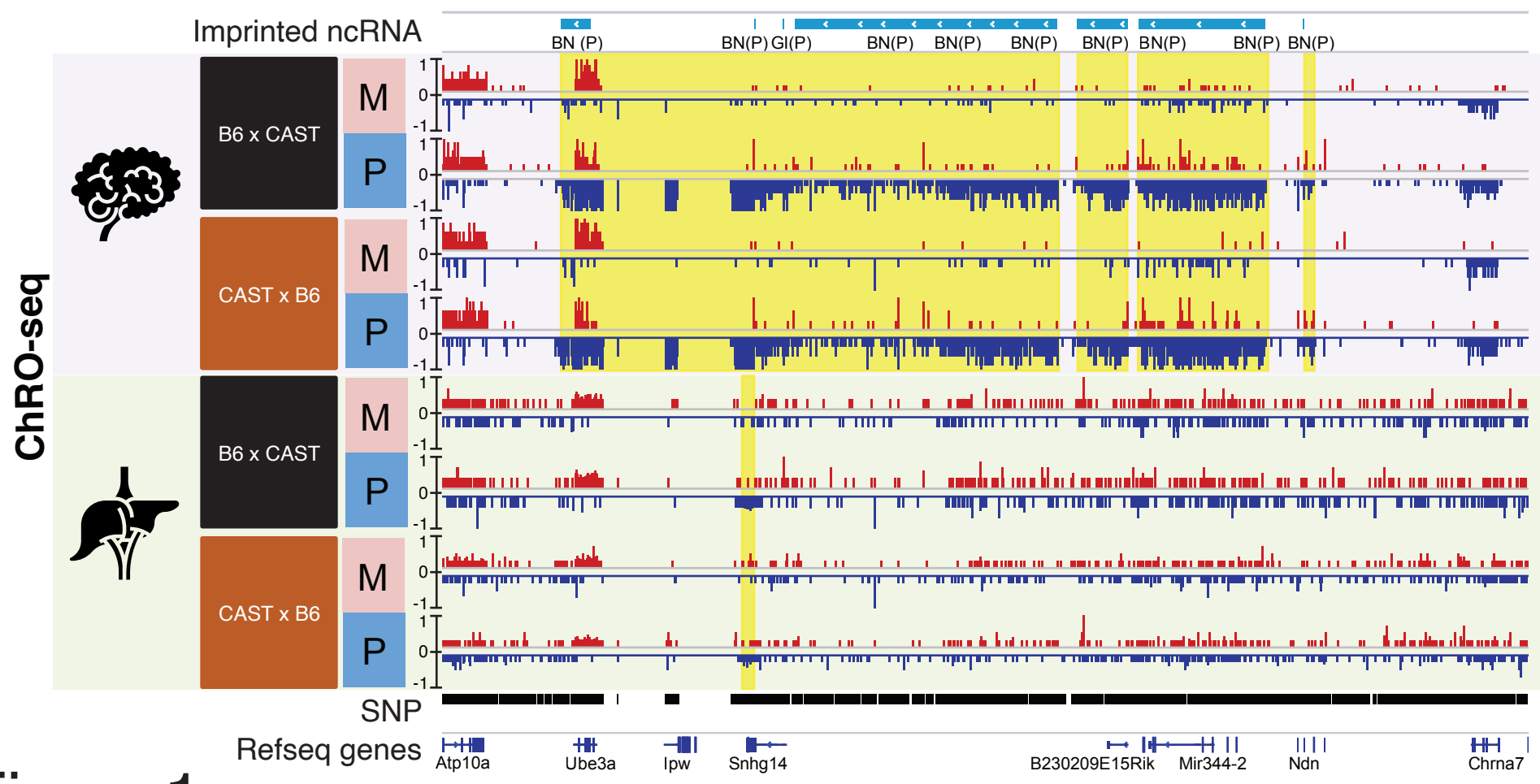

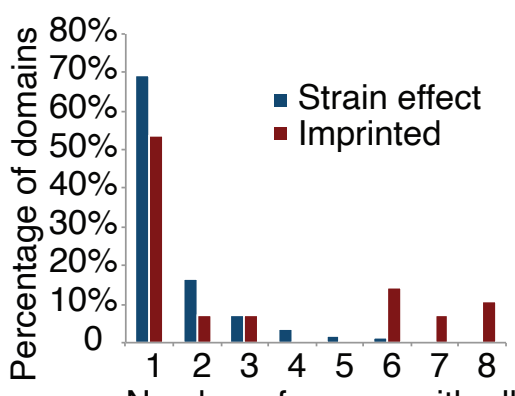

Number of organs with allelic biased blocks in the domain

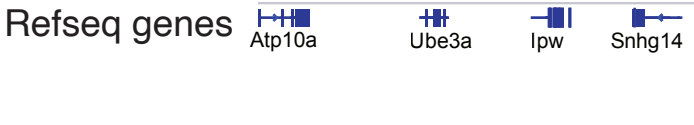

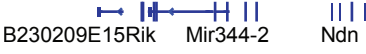


Figure 1: Reciprocal hybrid cross to understand the Pol II transcription cycle.

(A) Cartoon illustrates the reciprocal F1 hybrids cross design between the strains C57BL/6J (B6) and CAST/EiJ (CAST). We have seven independent crosses (3x C57BL/6 x Cast and $4 x$ Cast $x$ C57BL/6).

(B) Spearman's rank correlation of ChRO-seq signals in gene bodies. The color on the top indicates the direction of crosses: Black is B6 $\times$ CAST, brown is CAST $\times$ B6. The cartoon on the right indicates the organ each sample was harvested from.

(C) Cartoon depicts the methods used to identify allelic biased blocks, domains, and how they were classified as a strain-associated effect or imprinted.

(D) The pie chart shows the proportion of domains under strain effect or imprinted.

(E) The histogram shows the proportion of domains as a function of the number of gencode gene annotations in each domain.

(F) The browsershot shows an example of ChRO-seq data that has an imprinted domain (top row). The second and third rows show the imprinted protein-coding genes and imprinted non-coding RNA (ncRNA) from all organs. (BN:brain, LV:liver, SK:skeletal muscle, Gl: large intestine, HT: heart, KD: kidney, P: paternal, M: maternal). The yellow shade indicates the imprinted regions in the brain and liver.

(G) The histogram shows the proportion of domains as a function of the domain length.

$(\mathrm{H})$ The bar chart shows the percentage of domains as a function of the number of organs with allelic biased blocks in the domain. 
bioRxiv preprint doi: https://doi.org/10.1101/2021.05.23.445279; this version posted May 30, 2021. The copyright holder has placed this

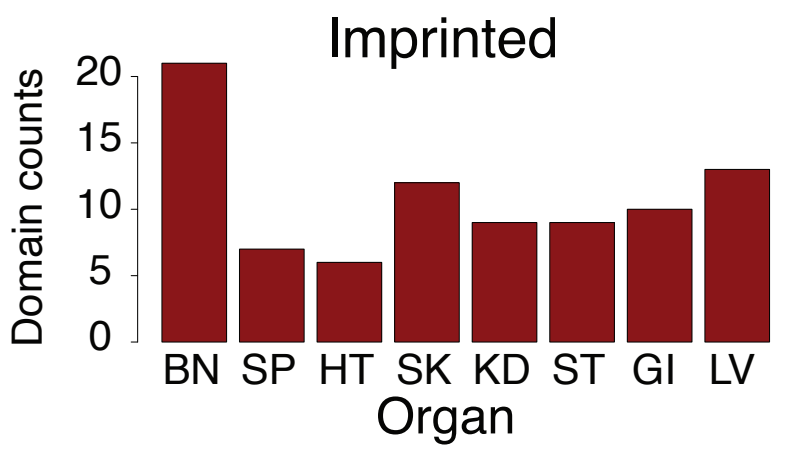

\section{Imprinted}
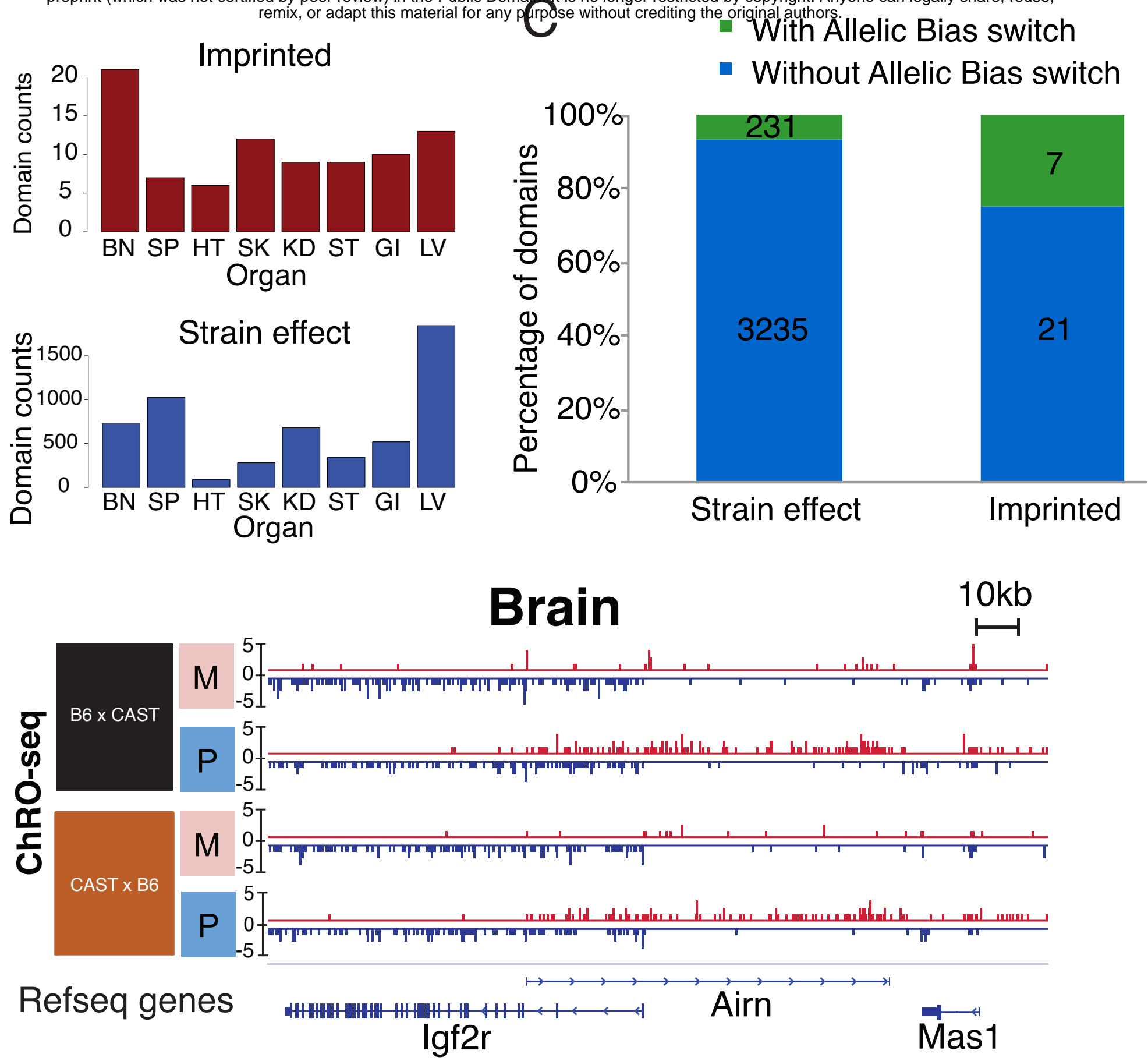

\section{Figure2}

Figure 2: Features associated with imprinting domains.

(A) The bar charts show the number of imprinted (top) and strain effect domains (bottom) in each organ. (BN:brain, SP: spleen, HT: heart, SK:skeletal muscle, KD: kidney, ST:stomach, GI: large intestine, LV:liver)

(B) The browsershot shows allele-specific ChRO-seq signal from the Igf2r locus in brain, which contains the imprinting-associated ncRNA, Airn. M: maternal-specific reads; P: paternal-specific reads.

(C) Stacked bar charts show the percentage of strain effect and imprinted domains that contain transcription units with opposite allelic bias (called switches). Those without allelic bias switches were consistently biased to the same direction (for imprinted domain, all maternally-biased or all paternally-biased, no switch). Those domains with allelic bias switches contain blocks biased to different directions (some maternally-biased and some paternally-biased, with switches). 
Wang et al., 2011). We noted that genomic imprinting was frequently stronger in the brain than other adult organs analyzed here. Although imprinting was less likely to be organ-specific than strain-effect domains (Fig. 1H), those which were organ specific were largely imprinted in brain (Fig 2A). Moreover, of the domains that were imprinted in 6 or more organs ( $n=9$ of $28 ; \sim 33 \%$ ), the imprinting domain was generally larger in the brain than in other organs, consistent with previous observations (Andergassen et al., 2017; Plasschaert and Bartolomei, 2015; Wilkins, 2014). An outstanding example was the Angelman and Prader-Willi syndrome locus, which was imprinted in multiple organs including brain, liver, heart, skeletal muscle, large intestine, and kidney. However, while imprinting only affected Snhg14 and Snurf in most organs, imprinting affected a much broader region in the brain, resulting in imprinting of additional protein coding genes, including Ube3a and Ipw (Fig. 1F). This finding may explain why both Angelman and Prader-Willi syndrome have symptoms associated with behavior (Ho and Dimitropoulos, 2010; Pelc et al., 2008). In another example, we noted the opposite parent-of-origin effect in the brain as observed in other organs. Grb10 was imprinted in multiple organs, but it was transcribed from the opposite allele in the brain (paternal-specific) than all other adult mouse organs (maternalspecific). Taken together, these observations show that the brain frequently has a unique pattern of genomic imprinting that is not observed in other somatic organs.

\section{Discovery of imprinted ncRNAs}

In several well-characterized cases, imprinting is initiated by a ncRNA, as elegantly illustrated at the Igf2r / Airn locus (Sleutels et al., 2002). ChRO-seq clearly defined the location of known ncRNAs, including Airn and Kcnq1ot1 that mediate genomic imprinting (Fig. 2B). We discovered new candidate ncRNAs at several additional imprinted loci, including in the Angelman and Prader-Willi syndrome locus (Fig. 1F). Frequently, ncRNAs like Airn had an opposite allelic bias as the nearby imprinted protein-coding genes. Similar patterns were observed for ncRNAs in the Angelman and Prader-Willi syndrome locus (chr7), in which ncRNAs across the locus were transcribed in the opposite parental allele as several of the imprinted genes (e.g., Ube3a). In another interesting example, two protein coding genes, Peg3 and Usp29, were transcribed with opposite allelic bias as another protein coding gene, Zim1, without a clear ncRNA within the locus. Overall, 7 of the $28(25 \%)$ imprinted domains had transcripts with an opposite allelic bias occurring within the same domain, which reflects a significantly higher proportion than the $7 \%$ strain effect domains (Fisher's exact test, Odds ratio $=4.66, P=0.002$, Fig. $2 \mathrm{C}$ ). We note allelic differences in expression noted here are similar to well characterized examples, such as in X-chromosome inactivation, in which a ncRNA (Xist) is transcribed from the inactive copy of the $X$ chromosome(Augui et al., 2011). These differences may reflect the effect of nascent RNA transcription on the chromatin architecture of the locus, in a manner reminiscent of Airn, and possibly Xist.

\section{Widespread genetic changes in transcription initiation}

To begin dissecting the genetic basis for each stage of the transcription life cycle, we first focused on defining allele specific patterns of transcription initiation. The 5' end of nascent RNA, denoted by the 5 ' end of paired-end ChRO-seq reads, marks the transcription start nucleotide (TSN) of that nascent RNA (Kwak et al., 2013; Tome et al., 2018). We identified TSNs in which at least 5 unique reads share the same 5 ' end inside of regions enriched for transcription initiation 
identified using dREG after merging all samples from the same tissue (Wang et al., 2018). Using a recently described hierarchical strategy (Tome et al., 2018), we grouped candidate TSNs into transcription start sites (TSSs) and defined the maximal TSN as the position with the maximum 5' signal within each TSS (Fig. 3A). To verify that our pipeline identified transcription start nucleotides accurately, even in the absence of enzymatic enrichment for capped RNAs, we examined whether candidate transcription initiation sites were enriched for the initiator DNA sequence element, a defining feature of Pol II initiation (Kaufmann and Smale, 1994; Smale and Baltimore, 1989). Most organs, especially brain and liver (which were the most deeply sequenced and are the focus of the analysis below unless otherwise specified), had high information content showing the initiator element at maxTSNs (Supplementary Fig. 2A). Moreover, the relationship between read depth and TSNs was similar to those recently reported in human cells (Tome et al., 2018) (Supplementary Fig. 2B).

Allelic changes in transcription initiation frequency were common, occuring in $\sim 3-7 \%$ of different TSSs $(n=1,109-5,793$; binomial test FDR $<0.1)$. Changes in the frequency of initiation from TSSs predominantly reflect changes in the rates of transcription initiation at that gene. These changes likely reflect allelic differences in transcription factor binding and other regulatory processes that have been explored extensively elsewhere (Battle et al., 2014; Chen et al., 2016; Lappalainen et al., 2013; Montgomery et al., 2010; Pickrell et al., 2010).

To better define how DNA sequence shapes the precise genomic coordinates of transcription initiation, we focused on allele-specific changes in the position of TSNs within TSSs. We identified 1,006 (brain) and 1,389 (liver) TSSs in which the shape of the 5' end of mapped reads within that TSS changed between alleles (FDR $<=0.1$; Kolmogorov-Smirnov [KS] test) (see examples in Fig. 3B-C). As TSSs are typically regions spanning $\sim 80$ bp that are comprised of multiple TSNs (Carninci et al., 2006; Tome et al., 2018), which could have distinct patterns of allelic imbalance, we divided changes in TSS shape into two classes: cases that were driven predominantly by large changes in the abundance of Pol II at a single TSN position and cases in which multiple TSNs across the TSS contributed to changes in shape (see Methods). For example, the TSS giving rise to the transcription unit upstream and antisense to Rps6kc1 had major differences in just one of the TSNs (Fig. 3B, arrow head), whereas the promoter of Zfp719 has multiple changes in TSNs within the same TSS (Fig. 3C, arrow heads). In both examples, allelic changes result in differences in the position of the max TSN between alleles. Each of these two classes comprised approximately half of the changes in TSN shape in all organs examined here (Fig. 3D).

We examined how SNPs influence the precise location of transcription initiation between alleles. We compared the distribution of SNPs at allele specific TSSs to a control set composed of non allele-specific TSSs, which was designed to control for the ascertainment biases associated with having a tagged SNP in each allelic read. Single position driven allele-specific TSSs were associated with a strong, focal enrichment of SNPs around maxTSNs while the multiple position driven allele specific TSSs had a weaker, broader enrichment of SNPs (Fig. 3EF [liver] and Supplementary Fig. 2C-D [brain], yellow shade indicates FDR $<=0.05$; Fisher's exact test). SNPs within $5 \mathrm{bp}$ of the initiation site explain up to $\sim 15-20 \%$ of single-base driven allele specific transcription start sites (Fig. 3E and Supplementary Fig. 2C). This was predominantly explained by SNPs at the transcription start site itself, with an A highly enriched in the allele with highest max TSN usage at that position, consistent with the sequence preference 
bioRxiv preprint doi: https://dprorg/10.1101/2021.05.23.445279; this version posted May 30, 2021. The copyright holder has placed this

A preprint (which was not certified by geer1keview) intithe Public Domain. It is no longer restricted by copyright. Anyone can legally share, reuse,

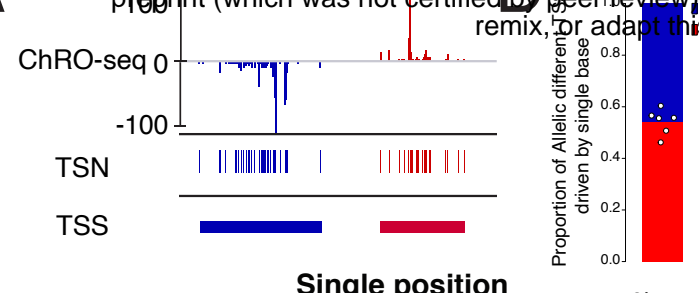

is isingaterial for any purpose without crediting the original authors.

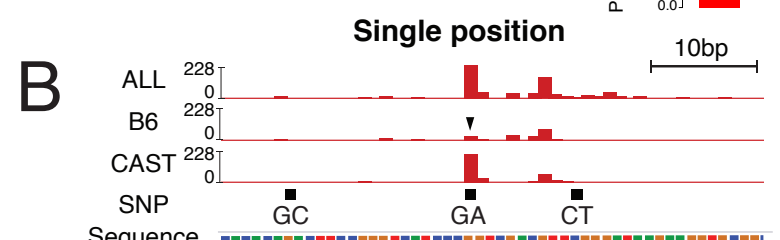

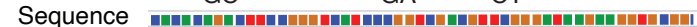

Refseq gene

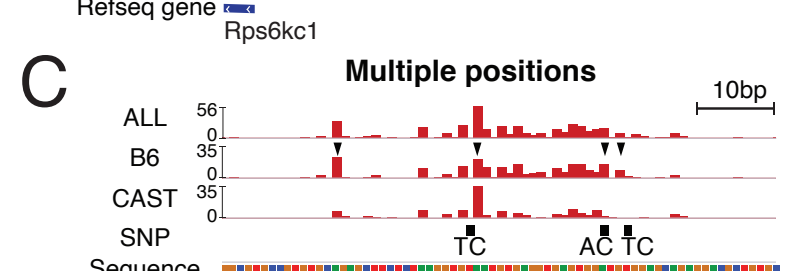

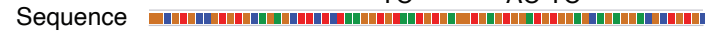
Refseq gene $\longrightarrow \overrightarrow{\text { Zfp719 }}$

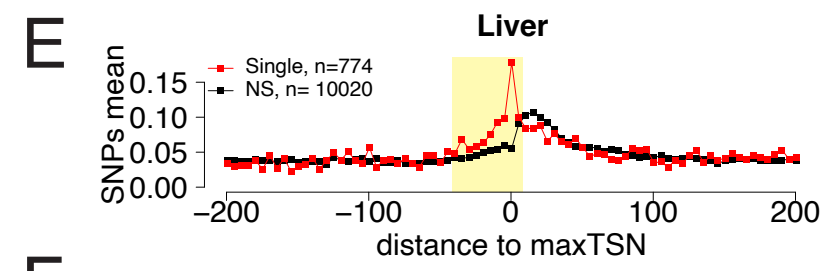

$\mathrm{F}$
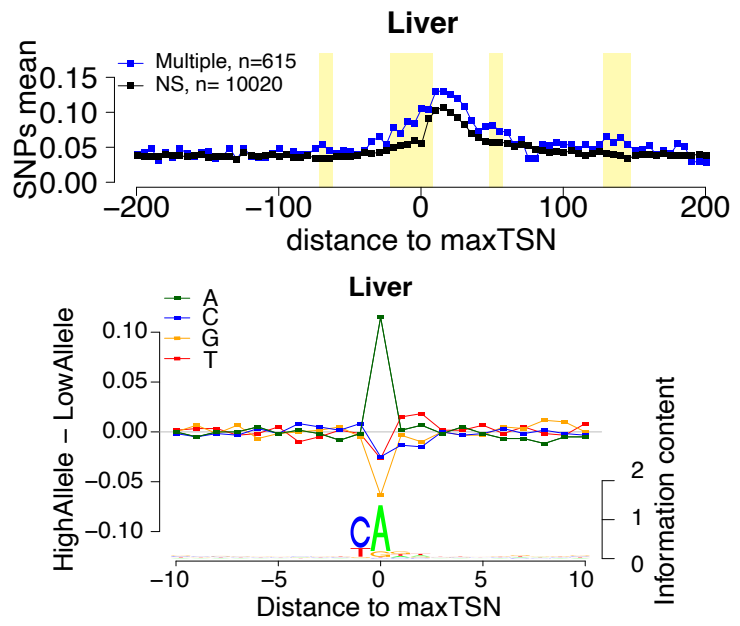

$\mathrm{H}$

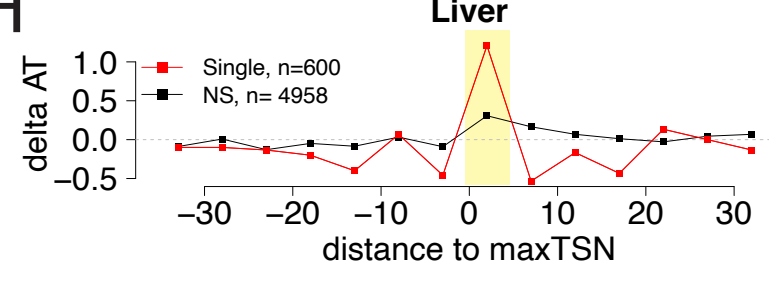

\section{Figure 3}


Figure 3: Allele specific effects on the distribution of transcription initiation.

(A) The ChRO-seq signals of the 5' end of the nascent RNA were used to define transcription start nucleotides (TSNs), or the individual bases with evidence of transcription initiation. TSNs within 60bp were grouped into transcription start sites (TSSs).

(B) The browsershot shows an example of allelic differences in the shape of TSS that are predominantly explained by a single TSN position (arrowhead).

(C) The browsershot shows an example of allelic differences in TSS driven by multiple TSNs within the same TSS, arrowheads indicate several prominent positions with allelic differences in Poll abundance.

(D) Stacked bar chart shows the average proportion of allelic differences in TSSs driven by a single TSN (red). The specific proportion for each of the six organs are shown by dots. Six organs were selected that showed a strong signal of Inr motif at the maxTSNs (brain, heart, liver, large intestine, stomach, and spleen).

(E) Scatterplot shows the average SNP counts as a function of distance to the maxTSN at sites showing allelic differences in TSSs driven by a single base in the liver. Red denotes changes in TSS shape (Kolmogorov-Smirnov (KS) test; FDR <=0.10); black indicates TSSs without evidence for differences in TSS shape (KS test; FDR > 0.90). Dots represent non-overlapping $5 \mathrm{bp}$ bins. Yellow shade indicates statistically significant differences (false discovery rate corrected Fisher's exact on 10 bp bin sizes, FDR <= 0.05)

(F) Scatterplot shows the average SNP counts as a function of distance to the maxTSN at sites showing allelic differences in TSS driven by multiple bases in the liver sample. Blue denotes changes in TSS shape classified as multiple TSN driven (Kolmogorov-Smirnov (KS) test; FDR <= 0.10); black indicates TSSs without evidence for differences in TSS shape (KS test; FDR > 0.90). Dots represent non-overlapping 5 bp bins. Yellow shade indicates statistically significant differences (false discovery rate corrected Fisher's exact on $10 \mathrm{bp}$ bin sizes, FDR <=0.05)

(G) The scatterplot shows the average difference in base composition between the allele with high and low TSN use around the maxTSN in single-base driven allele specific TSSs. The sequence logo on the bottom represents the high allele in single-base driven allele specific TSSs. The high/low allele were determined by the read depth at maxTSN.

$(\mathrm{H})$ The scatter plots shows the difference of AT contents between the high and low alleles with the maxTSN and -1 base upstream maxTSN masked. Dots represent $5 \mathrm{bp}$ non-overlapping windows. Red denotes single base driven allele specific TSSs; black denotes control TSSs with no evidence of allele specific changes. The yellow shade indicates a significant enrichment of AT(at high allele) to GC (at low allele) SNPs at each bin (size=5bp; Fisher's exact test, FDR $<=0.05$ ). 
of the initiator motif (Fig. 3G). By contrast, the enrichment of $C$ in the -1 position of the initiator motif was much weaker than the $A$ at the 0 position. Although this result may partially be explained by a bias in which the $C$ allele does not tag nascent RNA, it was consistent between organs (Supplementary Fig. 2E) and controlled based on the composition of the background set. Thus, our results suggest that the $A$ in the initiation site may be the most important genetic determinant of transcription initiation. Changes in TSS structure driven by multiple, separate TSNs, were enriched throughout the $\sim 30$ bp upstream, and to some extent downstream, potentially implicating changes in core transcription factor binding motifs or sequence specific transcription factors that influence the precise initiation site (Fig. 3F and Supplementary Fig. 2D).

Next we examined other factors near the TSS, aside from DNA within the initiator motif itself, that contributed to single base driven TSN choice. We noticed a higher frequency of $A$ and $T$ alleles downstream of the initiator motif on the allele with a higher max TSN (Fig. 3G). We hypothesized that the lower free energy of base pairing in A and T alleles would make them easier to melt during initiation, and could therefore increase the frequency of TSN usage at these positions. Indeed, a more direct examination of AT content in $5 \mathrm{bp}$ windows near the maxTSN identified a significantly higher AT content on the allele with the highest max TSN after masking DNA at positions -1 and 0 to avoid confounding effects of the initiator element (Fig. $3 \mathrm{H}$ and Supplementary Fig. 2F). This enrichment of high AT content was consistent in both brain and liver tissue, but was unique to single base driven max TSNs (Supplementary Fig. 2G-H). We conclude that multiple aspects of DNA sequence, including both sequence motif composition and the energetics of DNA melting, influence TSN choice in mammalian cells.

\section{Models of stochastic search during transcription initiation}

Next we examined how SNPs that affect a particular TSN impact initiation within the rest of the TSS. In the prevailing model of transcription initiation in S. cerevisiae, after DNA is melted, Pol II scans by forward translocation until it identifies a position that is energetically favorable for transcription initiation (Braberg et al., 2013; Kaplan et al., 2012; Qiu et al., 2020) (Fig. 4A). In mammals, Pol II is not believed to scan, but rather each TSN is believed to be controlled by a separate PIC (Luse et al., 2020). We considered how mutations in a strong initiator dinucleotide (CA) would affect transcription initiation under each model. Under the yeast model, we expected $\mathrm{CA}$ mutations to shift initiation to the next valid initiator element downstream. Under the mammalian model, we expected each TSN to be independent and therefore a mutation in the TSN would have no effect on the pattern of nearby initiation sites.

We analyzed 277 and 372 TSNs in brain and liver, respectively, where the high allele contained a CA dinucleotide while the other allele did not. Candidate initiator motifs within 20 bp of the $\mathrm{CA} /$ non-CA initiation site had slightly more initiation signal on the non-CA allele compared with the CA allele, consistent with the shooting gallery model but inconsistent with the prevailing model of independent TSNs expected in mammals (Fig. 4B; purple). By contrast, TSNs where both alleles contained a CA dinucleotide ( $n=8,147$ [brain] and 10,113 [liver]) did not show this same effect (Fig. 4B; gray). The difference was found for both adjacent CA dinucleotides and for weaker candidate $(\mathrm{Py})(\mathrm{Pu})$ initiator elements, and was consistent across both single-base and multiple-base TSS configurations (Supplementary Fig. 3). Unexpectedly, we also observed consistently higher initiation signals on the non-CA allele both upstream and downstream of the initiator dinucleotide (Fig. 4C). The signal for an increase on the non-CA allele stretched up to 
bioRxiv preprint doi: https://doi.org/10.1101/2021.05.23.445279; this version posted May 30, 2021. The copyright holder has placed this preprint (which was not certified by peer review) in the Public Domain. It is no longer restricted by copyright. Anyone can legally share, reuse, remix, or adapt this material for any purpose without crediting the original authors.

\section{A}

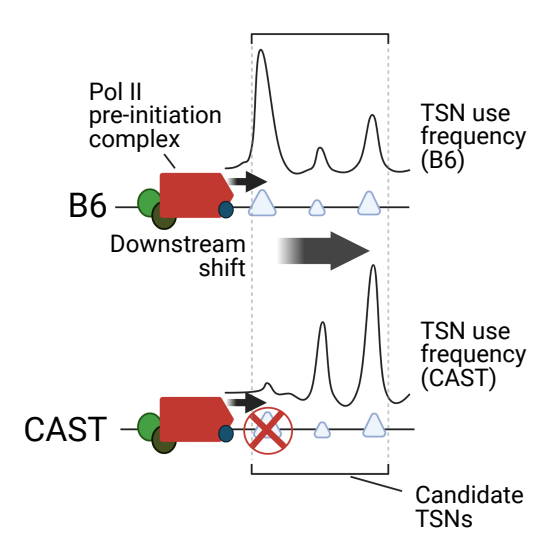

$\mathrm{B}$

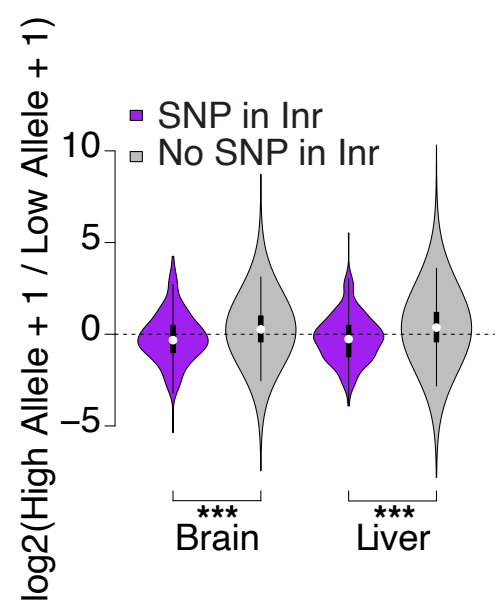

C

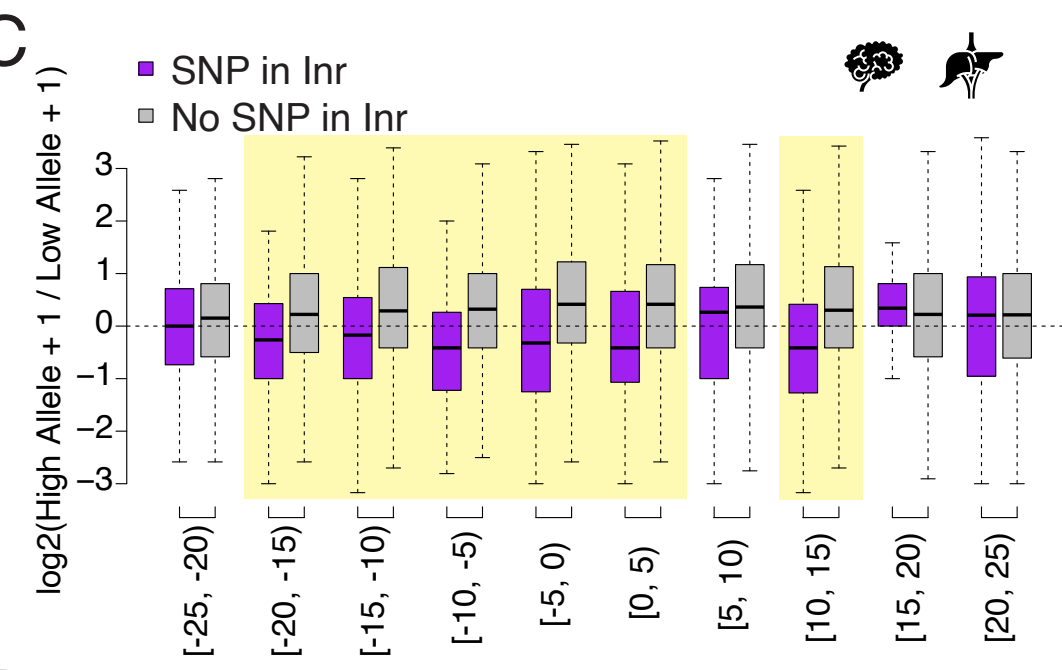

D

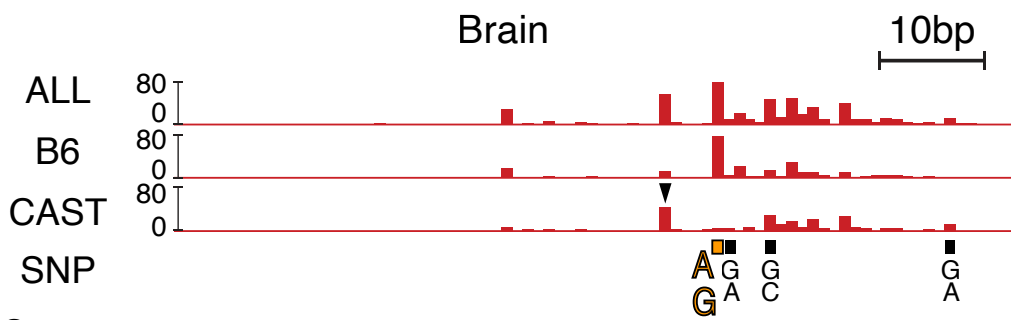

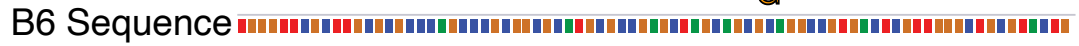


bioRxiv preprint doi: https://doi org/10.1101/2021.05.23.445279; this version posted May 30,2021 . The copyright holder has placed this

Figure 4: Browian motion model of transcription start nucleotide selection.

(A) Cartoon shows our expectation of the effects of allelic DNA sequence variation on transcription start nucleotide selection based on the "shooting gallery" model, in which Pol II initiates at potential TSNs (triangles) after DNA melting. We expected that mutations in a strong initiator dinucleotide (CA) on (for example) the CAST allele (bottom) would shift initiation to the initiator elements further downstream. The size of the triangle indicates the strength of the initiator.

(B) The violin plots show the distribution of ChRO-seq signal ratios on the candidate initiator motifs (including CA, CG, TA, TG) within 20bp of the maxTSNs that had a CA dinucleotide in the allele with high maxTSN (SNP in Inr, purple) or had a CA dinucleotide in both alleles (No SNP in Inr, gray). Note that the central maxTSN was not included in the analysis. Wilcoxon rank sum test with continuity correction is $p$-value $=5.665 \mathrm{e}-10$ for Brain and $p$-value $<2.2 e-16$ for liver.

(C) The box plots show the distribution of ChRO-seq signals ratios at TSNs with any variation of the initiator motif (including CA, CG, TA, TG) as a function of the distance from the maxTSNs that had a CA dinucleotide in the allele with high maxTSN (SNP in Inr, purple) or had a CA dinucleotide in both alleles (No SNP in Inr, gray). Yellow shade indicates Wilcoxon Rank Sum and Signed Rank Tests (SNP in Inr vs no SNP in Inr) with $\mathrm{fdr}<=0.05$. The TSSs were combined from the brain and liver samples.

(D) The browser shot shows an example of a maxTSN with increased initiation upstream and downstream of an allelic change in a CA dinucleotide. The orange block AG denotes a SNP at the maxTSN, in which B6 contains the high maxTSN with CA and CAST contains CG. The ChRO-seq signals at the alternative TSN with a CA dinucleotide (arrow head) upstream of the maxTSN were higher in the low allele (CAST in this case), resulting in a different maxTSN in CAST. 
$20 \mathrm{bp}$ both upstream and downstream of the CA/ non-CA dinucleotide. For instance, a SNP in the initiator element nearly abolished the dominant max TSN of the protein coding gene Smg9 in CAST (Fig. 4D, orange block). Instead, initiation in CAST shifted to a new maxTSN located upstream (Fig. 4D, arrow head), and also increased usage of several minor TSNs downstream (Fig. 4D). These findings are most consistent with a model in which DNA is melted to give Pol II access to the template strand, at which point Pol II scanning for an energetically favorable initiator element occurs in both directions by a stochastic process resembling brownian motion.

\section{Correspondence and disconnect between allele specific TSN and pause position}

We next examined allelic changes in the position of paused Pol II. To measure the position of the Pol II active site with single nucleotide precision, we prepared new ChRO-seq libraries in three organs (heart, skeletal muscle, and kidney from two female mice). New libraries were paired-end sequenced to identify the transcription start site and active site of the same molecule (Tome et al., 2018). New libraries clustered with those generated previously from the same organ (Supplementary Fig 4). Using the same pipeline we developed for transcription initiation, we identified regions enriched for transcription initiation and pausing, and validated that candidate maxTSNs were enriched for the initiator motif (Supplementary Fig. 5A). Our analysis identified 2,260 dREG sites with candidate changes in the shape of paused Pol II, assessed using the position of the Pol II active site, defined as the 3' end of RNA insert (FDR $<=0.1$; KolmogorovSmirnov [KS] test; see Methods).

Previous work has shown a tight correspondence between the site of transcription initiation and pausing, with pausing occurring predominantly in the window 20-60 bp downstream of the TSN (Tome et al., 2018). As expected, allelic changes in the position of paused Pol II were often coincident with changes in transcription initiation (Fig. 5A), particularly when the changes were large. In addition to the main component of correlation between initiation and pausing, however, we also identified changes in both pause and initiation that were independent of the other step in the transcription cycle. In at least 111 cases $(\sim 31 \%$ of sites where paused Pol II changed shape, and the single RNA molecule was tagged by a SNP), the position of the pause was identical between alleles, but the position of the max TSN that initiated the paused Pol II changed by 1-32 bp (Fig. 5B, top). Thus, in many cases where Pol II paused the same position in both alleles, the RNA molecules were initiated at distinct TSNs.

More commonly ( $n=269 ; \sim 52 \%$ of tagged RNA molecules with putative changes in pause shape), changes in Pol II pausing occurred between alleles despite being initiated from identical TSNs (Fig. 5B, bottom). Although more frequent, changes in the position of paused Pol II that shared the same TSN were slightly smaller in magnitude, typically $<10 \mathrm{bp}$. These cases represent changes in the Pol II pause site without changes in the position of transcription initiation.

\section{DNA sequence determinants of promoter proximal pause position}

To understand the genetic determinants of pausing, we analyzed the cases in which the same TSN had different maximal pause sites in the CAST and B6 alleles. As tagged SNPs in the window between the initiation and pause site were relatively rare, we increased our statistical power by analyzing all three of the organs together after removing duplicate initiation sites when they overlapped. After filtering, we identified 269 candidate positions in which the same TSN gave 
rise to separate pause distributions on the B6 and CAST alleles. As a control, we used 1,396 TSN/ pause pairs that were tagged by SNPs but did not have allelic changes in the pause position.

We first examined how short insertions or deletions affect the position of paused Pol II. Paused Pol II is positioned in part through physical constraints with TFIID, a core component of the pre-initiation complex (Fant et al., 2020). As a result of such connections with the pre-initiation complex, short insertions or deletions affecting the distance between the transcription start nucleoside and the maximal pause site altered the frequency of pausing at a model gene (D. melanogaster HSP70; (Kwak et al., 2013)). In our dataset, changes in pausing were highly enriched for small insertions and deletions between the maxTSN and pause site $(\mathrm{n}=56(21 \%)$; expected $=22(8 \%) ; p<1$ e-5, Fisher's exact test). Changes in pause position were correlated with changes in the size of the insertion or deletion, such that the distance between the max TSN and the pause site in the native genome coordinates was typically less than $5 \mathrm{bp}$ (Fig. 5C). This finding supports a model in which paused Pol II is placed in part through physical constraints with the pre-initiation complex (Fant et al., 2020; Kwak et al., 2013).

Next we identified single nucleotide changes that affect the position of the pause site. Previous studies have defined a C nucleotide at the paused Pol II active site (Gressel et al., 2017; Tome et al., 2018), which we recovered by generating sequence logos of max pause positions in our three murine organs (Fig. 5D, bottom). Additionally, we also observed a $\mathrm{G}$ in the +1 position immediately after the pause, and a G/A-rich stretch in the $10 \mathrm{bp}$ upstream of the pause site that lies within the transcription bubble (Fig. 5D, bottom). The 10 bp upstream of the pause position had a higher $\mathrm{G}$ content and a lower $\mathrm{C}$ content than the two surrounding windows (Fig. 5E). Thus, our data show that Pol II pauses on the $\mathrm{C}$ position immediately after a G-rich stretch.

Allelic differences at the $C$ base had the strongest association with the pause, followed by the -2 and -3 G bases (Fig. 5D; Supplementary Fig. 5B). We also noted enrichment of SNPs downstream of the pause, especially in the +1 position, although the number of SNPs supporting these positions were small. We also noted that multiple independent SNPs were frequently found in the same TSN/ pause pair (observed $\mathrm{n}=10$ (3.7\%); expected $=1(0.36 \%) ; p<2 \mathrm{e}-5$; Fisher's Exact Test; Fig. 5F), suggesting that multiple changes in the weak DNA sequence motifs associated with pausing were more likely to affect the position of paused Pol II. Collectively, indels and SNPs identified as enriched in the analysis above explained $49 \%$ of allele specific differences in the pause position (Fig. 5F). Thus, the DNA sequence determinants of pause position are largely found either within the pause site, the transcription bubble of paused Pol II, or insertions or deletions between the pause and initiation site.

\section{Pol II pause position is driven by the first energetically favorable pause site}

We extended our analysis of allelic differences in pausing to determine how multiple candidate pause positions early in a transcription unit (TU) collectively influence the position of paused Pol II. As in the analysis above, we focused on the set of allelic differences in pause shape in which the two alleles have a distinct maximal pause position $(n=269)$. By definition, these TUs had different maximal pause positions on the two alleles: on one allele the distance between the TSN and the pause position is shorter (which we call the 'short allele'), and on the other the distance between the TSN and the pause is longer ('long allele') (see cartoon in Fig. 5G, middle). We found that the DNA sequence motif near the long pause position was similar on both alleles, recapitulating the $C$ at the pause site and an enrichment of Gs in the transcription bubble (Fig. 
bioRxiv preprint doi: https://doi.org/101 101/2021.05.23.445279; this version posted May 30, 2021 . (he copyright holder has placed this
prefris

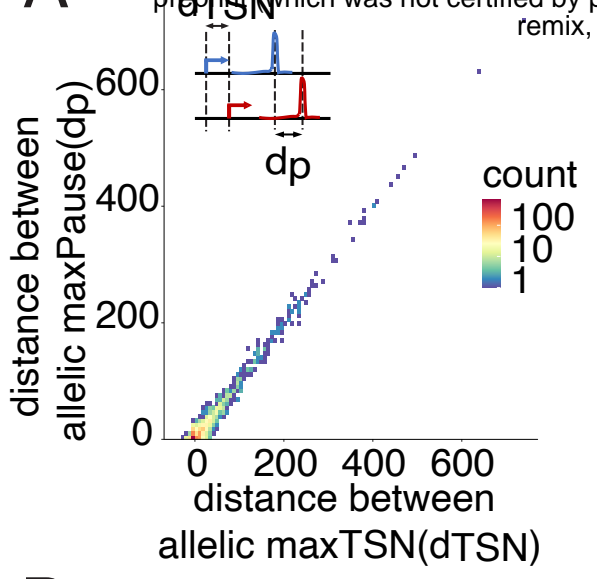

D

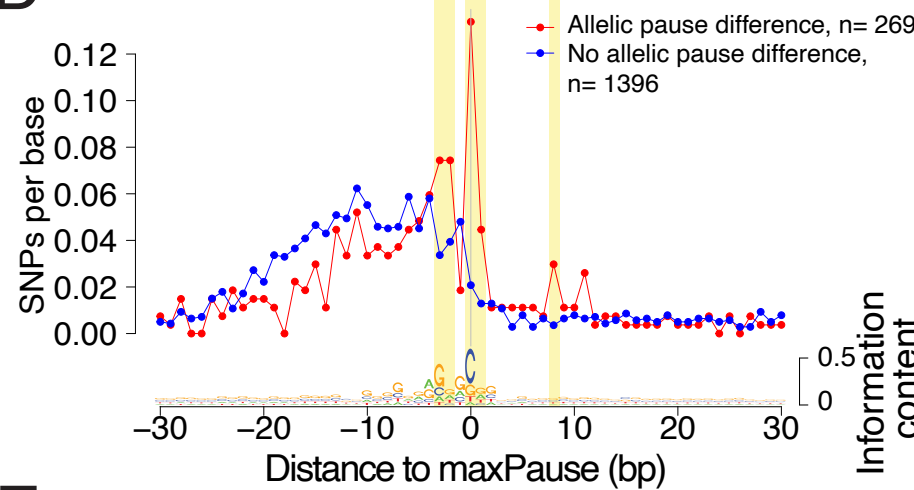

$E$
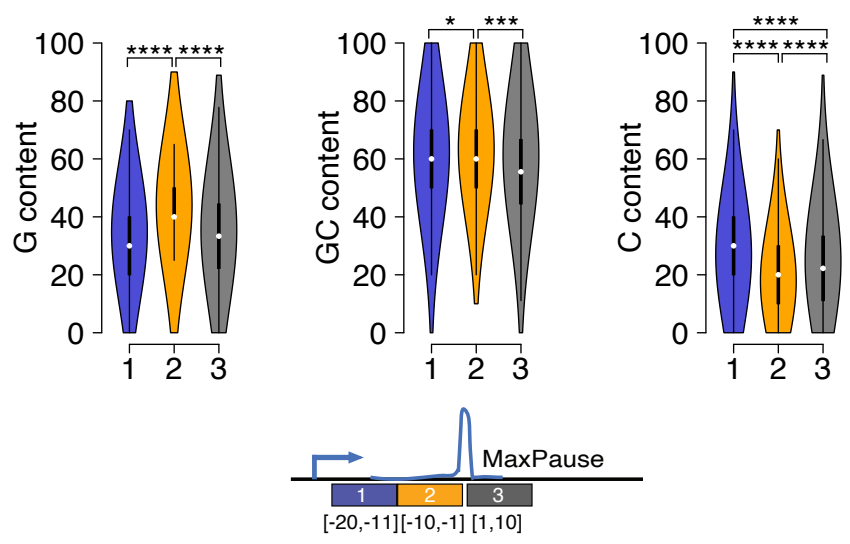

$\mathrm{H}$

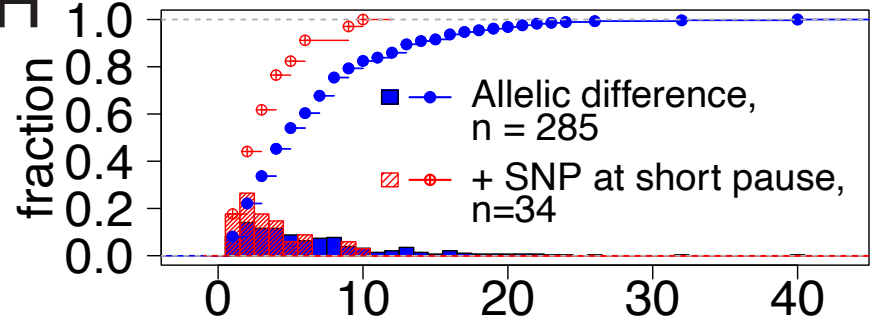

Distance between allelic maxPause

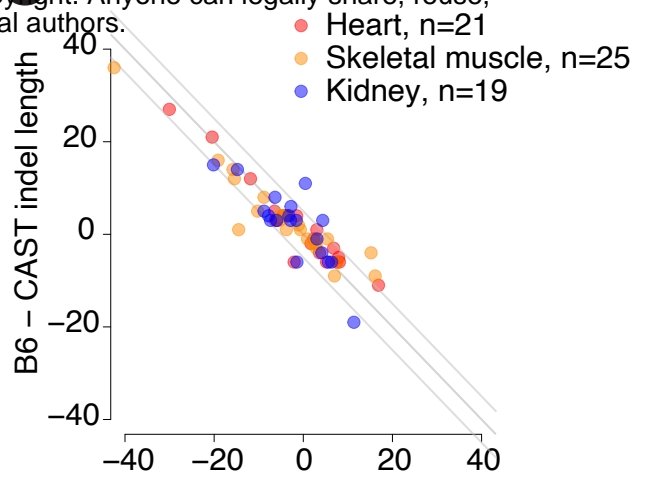

B6 - CAST average pause position on $\mathrm{mm} 10$
F Allelic pause difference No allelic pause difference
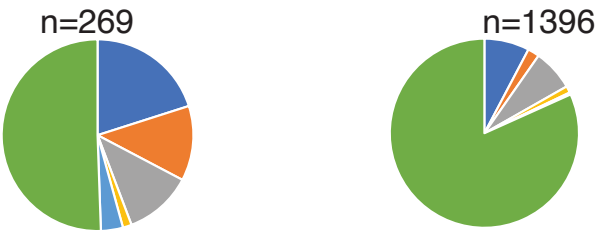

- indel SNPs at 0 from short pause - SNPs at -2 or -3

- SNPs at $+1 \quad$ Multiple events Other
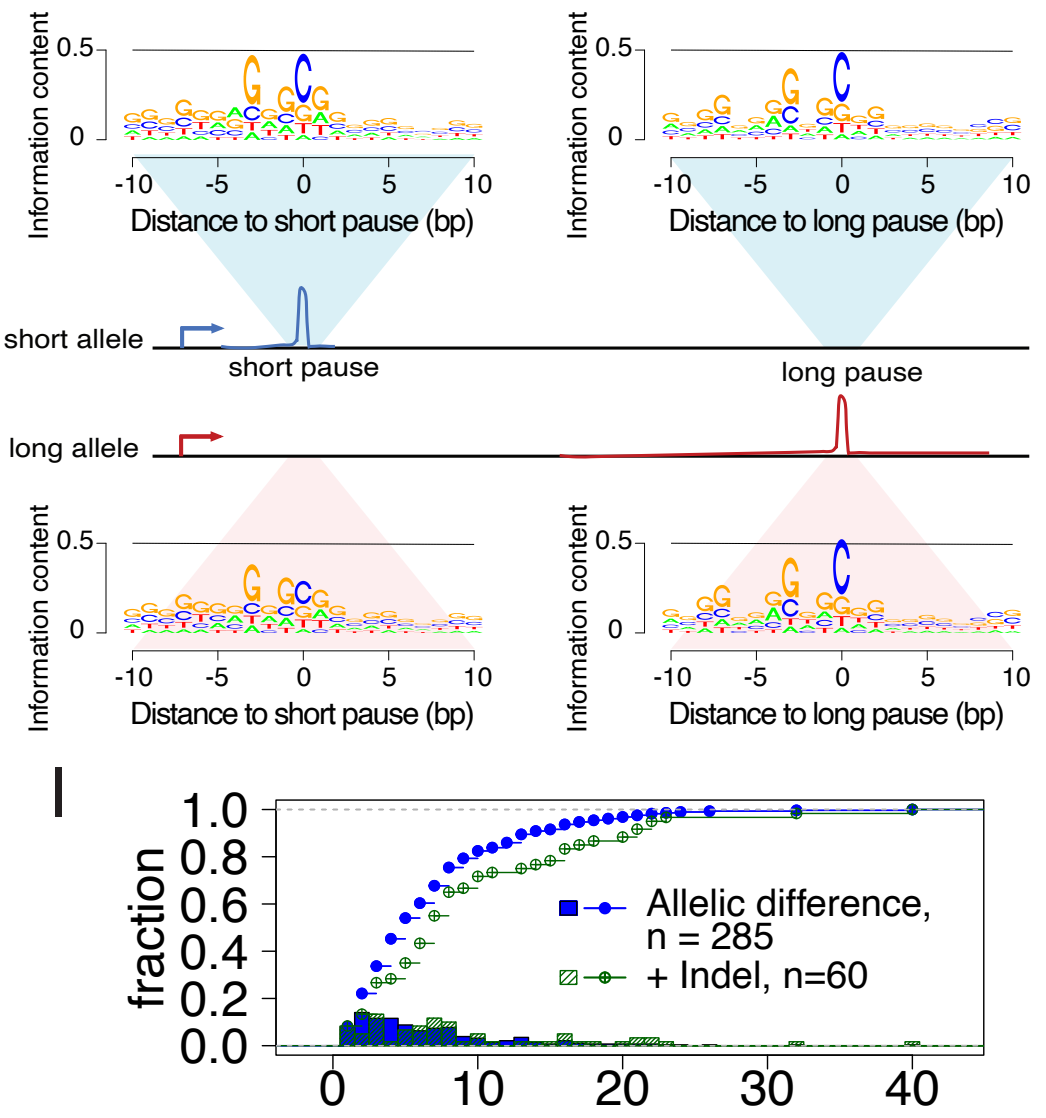

Distance between allelic maxPause

\section{Figure 5}


Figure 5: Allele specific effects on the distribution of Pol II in the promoter proximal pause.

(A) Scatterplots show the relationship between distances of allelic maxPause and allelic maxTSN within dREG sites with allelic different pause $(n=2,260)$.

(B) Top histogram shows the number of sites as a function of the distance between allelic maxTSN in which the allelic maxPause was identical $(n=359)$. Bottom histogram shows the number of sites as a function of the distance between allelic maxPause where the allelic maxTSN was identical $(n=823)$.

(C) Scatterplot shows the relationship between indel length and the allelic difference of the average pause position on the reference genome $(\mathrm{mm} 10)$. The pause positions of CAST were first determined in the CAST genome and then liftovered to mm10. Only sites initiated from the maxTSN and with allelic difference in pause shape were shown (KS test, $\mathrm{fdr}<=0.1$; also requiring a distinct allelic maximal pause). Color indicates the organs from which the TSN-pause relationship was obtained.

(D) Top: scatterplot shows the average SNPs per base around the position of the Pol II in which the distance between the maxTSN and the max pause was lowest (short pause). Red represents sites with allelic difference in pause shape (Allelic pause difference, KS test, $\mathrm{fdr}<=0.1$ with distinct allelic maxPause, $n=269$ ), Blue is the control group (No allelic pause difference, $\mathrm{KS}$ test, $\mathrm{fdr}>0.9$ and the allelic maxPause were identical, $\mathrm{n}=$ 1,396). Bottom: The sequence logo obtained from the maxPause position based on all reads ( $n=3,456$ max pause sites). Sites were combined from three organs, after removing pause sites that were identical between organs.

(E) Violin plots show the G content, GC content and C content as a function of position relative to maxPause defined using all reads (combined from three organs with duplicate pause sites removed, $n=3,456$ ), block 1 was 11 to $20 \mathrm{nt}$ upstream of maxPause, block 2 was 1 to $10 \mathrm{nt}$ upstream of maxPause, and block 3 was 1 to $10 \mathrm{nt}$ downstream of maxPause $\left({ }^{*} p<0.05,{ }^{* *} p<0.01,{ }^{* * *} p<0.001,{ }^{* * * *} p<0.0001\right)$. Block 2 had a higher $\mathrm{G}$ content and a lower $\mathrm{C}$ content than the two surrounding blocks.

(F) Pie charts show the proportion of different events around the maxPause of short alleles (short pause) with or without allelic pause differences. Sites were combined from three organs with duplicated pause sites removed.

(G) Sequence logos show the sequence content of short alleles and long alleles at 270 short pause sites and 278 long pause sites.

$(\mathrm{H})$ The histograms show the fraction of pause sites as a function of distance between allelic maxPause, i.e. the distance between the short and long pause. The lines show the cumulative density function. Blue represents pause sites with allelic differences $(n=$ $285)$; red is a subgroup of blue sites with a $C$ to $A / T / G$ SNP at the maxpause $(n=34)$. Two-sample Kolmogorov-Smirnov test $p$-value $=0.002694$

(I) The histograms show the fraction of pause sites as a function of distance between allelic maxPause. The lines show the cumulative density function. Blue is pause sites with allelic differences $(n=285)$, green is a subgroup of blue sites that contain indels between initiation and long pause sites $(n=60)$, Two-sample Kolmogorov-Smirnov test $\mathrm{p}$-value $=0.02755$. 
$5 G$, right). By contrast, DNA sequence changes affecting pause position occurred at the short pause position on the long allele (Fig. 5G; bottom left).

Our findings suggest a model in which single nucleotide changes that alter the free energy of the pause complex result in Pol II slipping to the next available position downstream. In favor of this model, when there was a SNP in the active site at the short pause, the max pause position moved downstream by $<10 \mathrm{bp}$, a relatively small amount compared to all changes in pause shape (Fig. 5H). By contrast, indels between the initiation and short pause site tended to have a larger effect on the allelic difference between pause positions (Fig. 5I). These observations support a model in which DNA sequence changes that disfavor pausing result in Pol II slipping downstream to the next pause site for which DNA sequence is energetically favorable, but maintaining physical connections that may exist between paused Pol II and the PIC.

\section{Allelic changes in gene length caused by genetic differences in Pol II termination}

AlleleHMM blocks (Chou and Danko, 2019), allelic biased blocks identified by AlleleHMM, were frequently found near the 3 ' end of genes, possibly reflecting allelic differences in Pol II termination that alter the length of primary transcription units. For example, Fam207a had an excess of reads on the CAST allele without a new initiation site that could explain allelic differences (Fig. 6A). To systematically identify allelic differences in the termination site, we identified AlleleHMM blocks that start inside of a protein coding transcription unit and end near or after the end of the same transcription unit (see Methods). Several lines of evidence suggest that these candidate allelic differences were enriched for bona-fide termination differences: the majority did not start near dREG sites, and overall the allele with higher expression tended to have similar ChRO-seq signal as the primary gene (Supplementary Fig. 6A).

To visualize ChRO-seq signal surrounding the window of allelic termination (AT window), we generated heat maps of ChRO-seq signal centered near the start of the allelic increase in expression and sorted by the length of the allelic termination window (Fig. 6B). Heatmaps showed a higher abundance of ChRO-seq reads in the allelic termination window on the allele with higher expression. Overall, we identified 317-931 candidates in each organ (total $n=3,450$ ). These allelic termination differences varied in size between 1kb and 100kb (Fig. 6C; Supplementary Fig. 6B).

Allelic differences in termination were consistent between different organs. An outstanding example is Fam207a, which has approximately the same allelic termination window in brain and liver (Fig. 6A). Furthermore, taking the subset of genes that were expressed and producing heat maps centered in the same position and the same order as in liver recovered similar patterns of allelic termination being evident in brain, spleen, or other organs (Fig. 6B). This result suggests that allelic differences in termination were driven predominantly by DNA sequence, with little effect from the trans environment.

Allelic differences in termination could be caused by factors that influence the mature mRNA, such as differences in the polyadenylation cleavage site (Mittleman et al., 2021, 2020). To determine whether allelic differences in termination correlate with differences in the composition of the mature mRNA, we sequenced poly-A enriched mRNA from two liver and brain samples. Genes that had allelic differences in termination frequently also had an AlleleHMM block identified in the mRNA-seq data (21-41\% of cases), potentially consistent with allelic differences in termination driving differences in the mature mRNA composition (Fig. 6D). In the majority of cases $(59-79 \%)$, we could not find any evidence that changes in allelic termination of the primary 
bioRxiv preprint doi: https://doi org/10.1101/2021.05.23.445279; this version posted May 30, 2021. The copyright holder has placed this preprint (which was not certified by peer review) in the Public Domain. It is no longer restricted by copyright. Anyone can legally share, reuse, remix, or adapt this material for any purpose without crediting the original authors.

transcript affected the mature mRNA composition. This indicates that many of the changes in Pol II termination do not affect the mature mRNA.

We examined whether changes in the mature mRNA composition were enriched for changes in mRNA stability. We estimated mRNA stability using the ratio of mRNA-seq to ChROseq signal (Blumberg et al., 2021). Our analysis did not find evidence that allelic changes in termination affecting the mature mRNA composition had a consistent effect on mRNA stability (Supplementary Fig. 6C), although we are likely underpowered to detect global changes in this analysis. Nevertheless, our data allowed us to conclude that allelic differences in Pol II termination are widespread and frequently do not affect mRNA composition or stability. 
bioRxiv preprint doi: https://doi.org/10.1101/2021.05.23.445279; this version posted May 30, 2021. The copyright holder has placed this preprint (which was not certified by peer review) in the Public Domain. It is no longer restrictodby copyright. Anyone can legally share, reuse, remix, or adapt this material for any purpose without crediting ho original aythors.
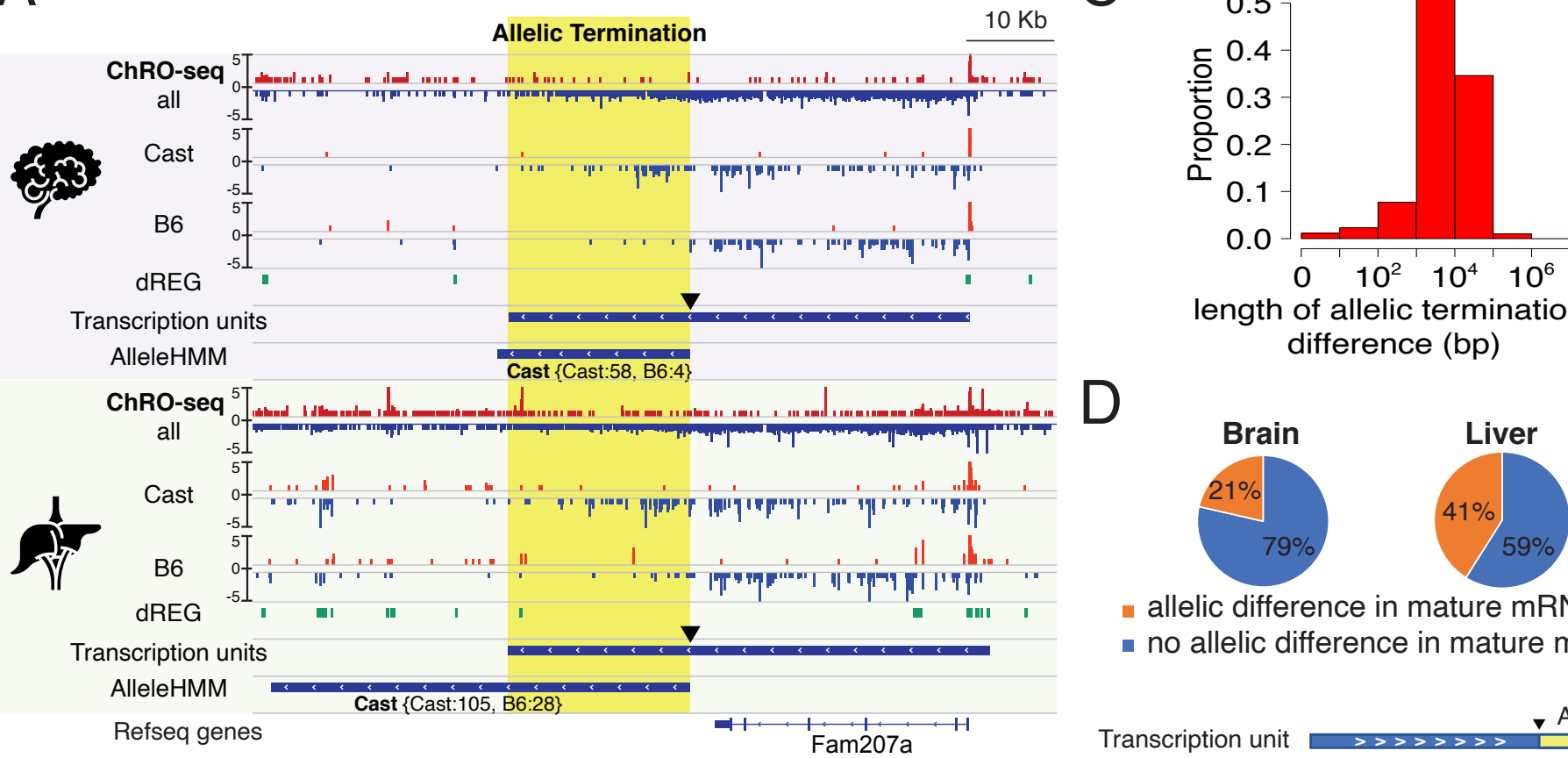

length of allelic termination difference (bp)

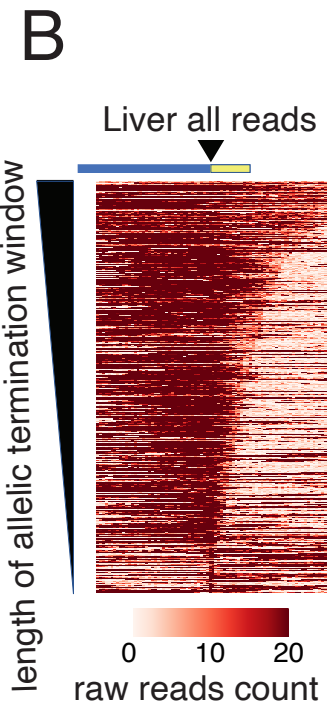

\section{Liver ChRO-seq}

Liver short allele Liver long allele
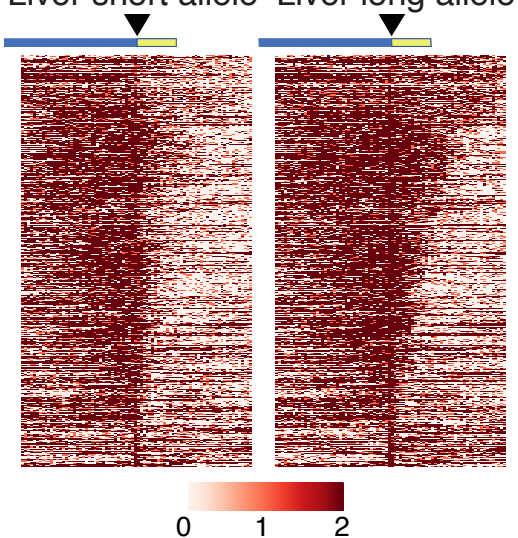

raw reads count
Brain ChRO-seq

Liver short allele Liver long allele

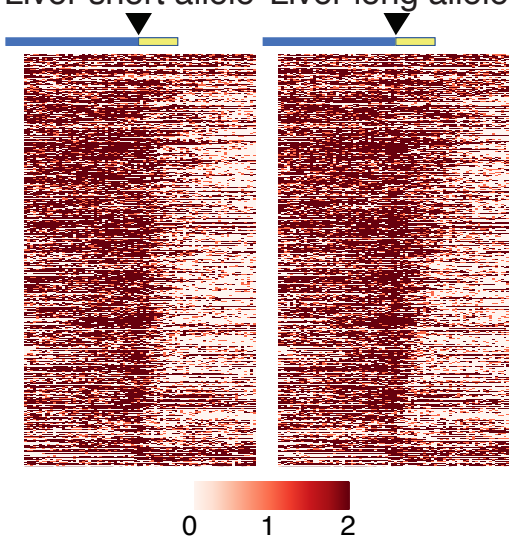

raw reads count
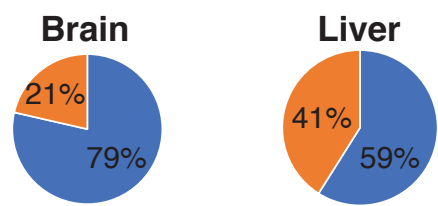

allelic difference in mature mRNA

- no allelic difference in mature mRNA
Transcription unit

AT window

\section{Spleen ChRO-seq}

Liver short allele Liver long allele

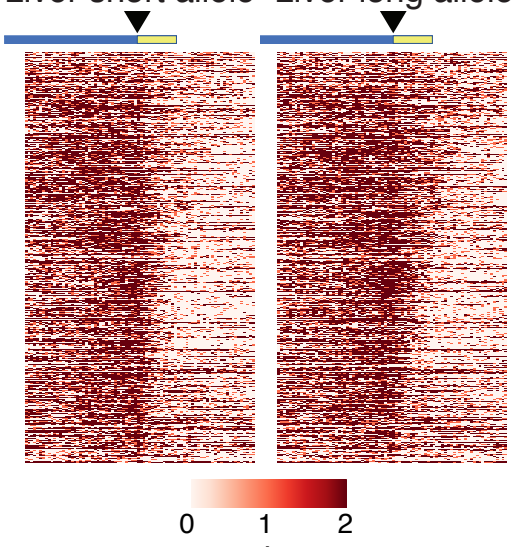

raw reads count

\section{Figure 6}

Figure 6: Widespread allele specific differences in the Pol II termination site.

(A) The browser shot shows an example of allelic termination differences (yellow shade) in both brain and liver. Pol II terminates earlier on the B6 allele, resulting in a longer transcription unit on the CAST allele. The difference in allelic read abundance was identified by AlleleHMM. We defined the allelic termination difference (yellow shade) using the intersection between the transcription unit and AlleleHMM blocks.

(B) Heatmaps show the raw read counts in transcription units (blue bar) with an allelic termination difference (yellow bar), centered at the beginning of allelic termination (solid triangle). The heatmap bin size is $500 \mathrm{bp}$, and $20 \mathrm{~kb}$ is shown upstream and downstream. The rows were sorted by the length of allelic termination differences determined by ChROseq signals from Liver. The short and long alleles were determined based on analysis of the liver.

(C) The histogram shows the fraction of transcription units as a function of the length of allelic termination difference .

(D) Pie charts show the proportion of transcription units with allelic termination difference that also contains allelic difference in mature mRNA (orange). 


\section{Discussion}

We examined how DNA sequence affects the precise, nucleotide position of Pol II during each stage of the transcription cycle. We generated and analyzed new ChRO-seq libraries which map the location and orientation of RNA polymerase in eight murine organs. Our study used an F1 hybrid cross between CAST and B6, which we contend is particularly well suited to this problem because experimental batch variation is identical between alleles. As a result, we can be confident in differences observed in our analysis, even in cases where the differences between the CAST and B6 alleles were relatively small in magnitude. Using this system, our analysis provides new insight into how DNA sequences shape the precise position and dynamics of Pol II. Here we discuss how our findings impact the prevailing models governing each stage in the Pol II transcription cycle.

DNA sequence changes within $\sim 5-10 \mathrm{bp}$ of the transcription initiation site frequently determine the relative use of that initiation site. The $A$ base at the transcription start site itself stood out as the most important determinant of transcription initiation. Changes in the cytosine nucleotide at the -1 position had surprisingly little evidence for differences in our analysis despite being as informative about initiation in the initiator sequence motif, though this may be impacted by the $\mathrm{A}$ at position 0 tagging the nascent RNA whereas the $\mathrm{C}$ does not. We also noted an enrichment of AT nucleotides surrounding the initiation site on the allele with high TSN usage. We speculate that enrichment of a relatively high AT composition may influence the initiation site because AT-rich DNA requires lower free energy to melt (Breslauer et al., 1986). Thus, the DNA sequence composition of the initiation site itself, as well as the sequence composition of surrounding nucleotides, impact transcription initiation.

Our results have also advanced models of transcription initiation. In the prevailing model of initiation in yeast, DNA is melted and Pol II scans by forward translocation until it identifies an energetically favorable TSN (Braberg et al., 2013; Kaplan et al., 2012; Qu et al., 2020). Mammals are not believed to scan, but rather independent PICs are believed to support initiation from a very narrow window (Luse et al., 2020). Intriguingly, DNA sequence changes in the initiator element affect the use of nearby initiation sites both upstream and downstream. We think the most straightforward interpretation of these results is that Pol II samples candidate initiation sites in both directions by a process resembling a one-dimensional brownian motion along the DNA. We note that an important assumption of our analysis is that the change in initiator sequence does not feed back and affect the DNA binding position of other components of the PIC. An alternative model that we are not able to discount completely is that feedback between transcription initiation and the binding site of other PIC components changes the site of DNA melting and leads to the PIC being assembled in nearby positions. We believe this interpretation is unlikely. Notably, the effect we report here appears confined to 20 bp of DNA surrounding the affected initiation site. It seems likely to us that any feedback would affect all TSNs within the TID, but this possibility does not appear to be supported by our analysis. In either case, however, it is clear from our data that changes in the DNA sequence of initiator elements tend to increase the use of candidate initiators nearby.

By analyzing allelic changes in pause position from the same initiation site, we have learned much about how DNA sequence influences the precise coordinates of promoter proximal pause. Our results show that the pause position occurs at a $C$ nucleotide downstream of a G-rich 
stretch, similar to motifs enriched at pause positions in prior studies (Gressel et al., 2017; Tome et al., 2018). As cytosine is the least abundant ribonucleotide (Traut, 1994), previous authors proposed it is the slowest to incorporate into nascent RNA, explaining its association with the Pol Il pause (Tome et al., 2018). In addition, we also identified a G-rich stretch that coincides with the position of the transcription bubble, as well as a guanine nucleotide just downstream of the pause position. The enrichment of $G$ nucleotides in the transcription bubble has a higher stability of RNADNA hybridization without using a cytosine nucleotide, and may serve to stabilize the RNA-DNA hybrid within the transcription bubble while Pol II remains paused.

We also noted widespread allelic differences in the site of transcription termination, which resulted in substantial differences in the length of primary transcription units between alleles. Allelic differences in transcription termination were largely similar between different organs, implicating DNA sequence differences as the major determinants of transcription termination. The majority $(60-80 \%)$ of allelic differences in termination did not affect the sequence composition of the mature mRNA, and when they did, we found no evidence for systematic differences in RNA stability. This may be influenced by purifying selection acting to remove many of the genetic variants with a large effect on mRNA.

In summary, our study dissects how DNA sequences impact steps during the Pol II transcription cycle. The dynamics of Pol II on each position of the genome can influence the rate of mRNA production and may impact organismal phenotypes. Our work is a first step in understanding the link between the core steps in transcriptional regulation and the impact they may have on phenotypes in humans and other animals. 


\section{Methods}

\section{Experimental Methods:}

Mouse experiments: The mice used in this study were reciprocal $\mathrm{F} 1$ hybrids of the strains C57BL/6J and CAST/EiJ. All mice were bred at Cornell University from founders acquired from the Jackson Laboratory. All mice were housed under strictly controlled conditions of temperature and light:day cycles, with food and water ad libitum. All mouse studies were conducted with prior approval by the Cornell Institutional Animal Care and Use Committee, under protocol 2004-0063.

Tissue collection: Mice were euthanized at 22 to 25 days of age by $\mathrm{CO}_{2}$, followed by cervical dislocation. All mice were euthanized between 10 a.m. and 12 p.m., immediately after removal from their home cage. Whole brain, eye, liver, stomach, large intestine, heart, skeletal muscle, kidney, and spleen were rapidly dissected and snap frozen in dry ice.

mRNA isolation/ RNA-seq library prep:RNA was extracted from the brain and liver of a male and a female mouse (both $22 \mathrm{~d}$ of age). Tissue samples were frozen using liquid nitrogen and pulverized using a mallet and a mortar. 100mg of each tissue was used for a TRIzol RNA extraction. Briefly, $1 \mathrm{~mL}$ of TRizol was added to each sample, chloroform was used for phase separation of the aqueous phase containing RNA, RNA was precipitated using isopropanol and washed with $75 \%$ ethanol. A total of $400 \mathrm{ng}$ of RNA was input into the RNA-seq library prep. Poly-A containing mRNA was enriched for 2 rounds using the NEBNext Poly $(A)$ mRNA Magnetic Isolation Module. Stranded mRNA-seq libraries were prepared by the Cornell TREx facility using the NEBNext Ultra II Directional RNA Library Prep Kit. Libraries were sequenced using an Illumina NextSeq500.

Chromatin isolation: Chromatin was isolated and ChRO-seq libraries were prepared following the methods introduced in our recent publication (Chu et al., 2018). Briefly, tissue was cryopulverized using a cell crusher (http://cellcrusher.com). Tissue fragments were resuspended in NUN buffer (0.3 M NaCl, $1 \mathrm{M}$ Urea, 1\% NP-40, $20 \mathrm{mM}$ HEPES, pH 7.5, $7.5 \mathrm{mM} \mathrm{MgCl2,} 0.2 \mathrm{mM}$ EDTA, 1 mM DTT, 20 units per ml SUPERase In Rnase Inhibitor(Life Technologies, AM2694), 1× Protease Inhibitor Cocktail (Roche, 11873580 001)).Samples were vortexed vigorously before the samples were centrifuged at $12,500 \mathrm{xg}$ for $30 \mathrm{~min}$ at $4 \mathrm{C}$. The NUN buffer was removed and the chromatin pellet washed with $1 \mathrm{~mL} 50 \mathrm{mM}$ Tris- $\mathrm{HCl}, \mathrm{pH} 7.5$ supplemented with 40 units of RNase inhibitor. Samples were centrifuged at $10,000 \times \mathrm{g}$ for 5 minutes at $4 \mathrm{C}$ and the supernatant discarded. Chromatin pellets were resuspended in storage buffer $(50 \mathrm{mM} \mathrm{Tris-HCl}$, pH 8.0, 25\% glycerol, $5 \mathrm{mM} \mathrm{Mg(CH3COO)2,} 0.1 \mathrm{mM}$ EDTA, $5 \mathrm{mM}$ DTT, 40 units per ml Rnase inhibitor) using a Bioruptor sonicator. The Bioruptor was used following instructions from the manufacturer, with the power set to high, a cycle time of $10 \mathrm{~min}$ (30s on and 30s off). The sonication was repeated up to three times if necessary to resuspend the chromatin pellet. Samples were stored at $-80 \mathrm{C}$. 
ChRO-seq library preparation: $\mathrm{ChRO}$-seq libraries were prepared following a recent protocol (Dig Bijay Mahat et al., 2016). We prepared some libraries to achieve single nucleotide resolution for the Pol II active site. In these cases, the chromatin pellet was incubated with $2 x$ run-on buffer (10 mM Tris-HCl, pH 8.0, 5 mM MgCl2,1 mM DTT, 300 uM KCl, 20 uM Biotin-11ATP (Perkin Elmer, NEL544001EA), 200 uM Biotin-11-CTP (Perkin Elmer, NEL542001EA), 20 uM Biotin-11-GTP (Perkin Elmer, NEL545001EA), 200 uM Biotin-11-UTP (Perkin Elmer, NEL543001EA)) for 5 minutes at $37 \mathrm{C}$. In some libraries we modified the run-on buffer to extend the length of reads for more accurate allelic mapping at the expense of single nucleotide resolution for the Pol II active site. In these cases, the run-on reaction was performed using a different ribonucleotide composition in the nuclear run-on buffer $(10 \mathrm{mM}$ Tris- $\mathrm{HCl}, \mathrm{pH} 8.0,5 \mathrm{mM}$ $\mathrm{MgCl} 2,1 \mathrm{mM}$ DTT, $300 \mathrm{mM} \mathrm{KCl}, 200 \mu \mathrm{M}$ ATP (New England Biolabs (NEB), N0450S), $200 \mu \mathrm{M}$ UTP, $0.4 \mu$ M CTP, $20 \mu$ M Biotin-11-CTP (Perkin Elmer, NEL542001EA), $200 \mu$ M GTP (NEB, N0450S)). The run-on reaction was stopped by adding Trizol LS (Life Technologies, 10296-010) and RNA was pelleted with the addition of GlycoBlue (Ambion, AM9515) to visualize the RNA. RNA pellet was resuspended in diethylpyrocarbonate (DEPC)-treated water. RNA was heat denatured at $65 \mathrm{C}$ for $40 \mathrm{~s}$ to remove secondary structure. RNA was fragmented using base hydrolysis (0.2N NaOH on ice for $4 \mathrm{~min})$. RNA was purified using streptavidin beads (NEB, S1421S) and removed from beads using Trizol (Life Technologies, 15596-026). We ligated a 3' adapter ligation using T4 RNA Ligase 1 (NEB, M0204L). We performed a second bead binding followed by a 5' decapping with RppH (NEB, M0356S). RNA was phosphorylated on the 5' end using T4 polynucleotide kinase (NEB, M0201L) then ligated onto a 5 ' adapter. A third bead binding was then performed. The RNA was then reverse transcribed using Superscript III Reverse Transcriptase (Life Technologies, 18080-044) and amplified using Q5 High-Fidelity DNA Polymerase (NEB, M0491L) to generate the ChRO-seq libraries. Libraries were sequenced using an Illumina HiSeq by Novogene. All adapter sequences and barcodes used for each sample are depicted in Supplementary Table 3 and Supplementary Fig. 7-8.

\section{Data analysis:}

\section{Read mapping, transcription start site, and transcription unit discovery:}

Processing and mapping ChRO-seq reads: Paired-end reads with single nucleotide precision were processed and aligned to the reference genome $(\mathrm{mm} 10)$ with the proseq2.0

(https://github.com/Danko-Lab/proseq2.0). Libraries in which we tailored the run-on to extend the length of reads were pre-processed, demultiplexed, and aligned to the reference genome (mm10) with the proseqHT_multiple_adapters_sequencial.bsh. AlleleDB (Chen et al., 2016; Rozowsky et al., 2011) align the $\mathrm{R} 1$ reads to the individual B6 and Cast genomes. In brief, the adaptor sequences were trimmed with the cutadapt, then PCR duplicates were removed using unique molecular identifiers (UMIs) in the sequencing adapters with prinseq-lite.pl (Schmieder and Edwards, 2011). The processed reads were then aligned with BWA (mm10) in analyses not using individual genome sequences (Li and Durbin, 2009), or with bowtie (Langmead et al., 2009) as input for AlleleDB. When bowtie was used, we selected either the R1 or R2 files for alignment for analyses requiring either the 5' or 3' end of the RNA insert. All scripts for mapping can be found publicly at: 
https://github.com/Danko-Lab/F1 80rgans/blob/main/00 F1 Tissues proseq pipeline.bash https://github.com/DankoLab/utils/blob/master/proseq HT/proseqHT multiple adapters sequencial.bsh

Processing and mapping RNA-seq reads: We used STAR (Dobin et al., 2013) to align the RNAseq reads. To avoid bias toward the $\mathrm{B} 6$ genome, we did not use any gene annotations for mapping, but used the list of splicing junctions generated by STAR. Mapping was performed in three stages: First, reads were first mapped without annotation and STAR generated a list of splicing junctions (sj1) from the data. Second, to identify potential allele specific splicing junctions, we performed allele specific mapping using STAR which takes as input a VCF file denoting SNPs differentiating Cast and B6, using the initial splice junction list (sj1). This personalized mapping was used to generate a more complete list of splice junctions (sj2). Third, we identified allele specific alignments by using the WASP option provided by STAR (van de Geijn et al., 2015). In this final mapping, we used the splice junction list (sj2) and a VCF file. This procedure generated a tagged SAM file ( $\mathrm{vW}$ tag) providing the coordinates of allele specific alignments and their mapping position. Scripts can be found here: https://github.com/DankoLab/F1 80rgans/blob/main/termination/F1 RNAseq forManuscript.sh

dREG: For each organ, we merged all reads from each replicate and cross to increase the power of dREG. BigWig files representing mapping coordinates to the $\mathrm{mm} 10$ reference genome were uploaded to the dREG web server at http://dreg.dnasequence.org (Wang et al., 2018). All of the output files were downloaded and used in subsequent data analysis. Scripts used to generate the BigWig files can be found at: https://github.com/Danko-Lab/F1 80rgans/blob/main/F1 TSN Generate BigWig.sh

Transcript unit prediction using tunits: We used the tunit software to predict the boundaries of transcription units de novo (Danko et al., 2018). We used the 5 state hidden Markov model (HMM), representing background, initiation, pause, body, and after polyadenylation cleavage site decay from tunits. To improve sensitivity for transcription unit discovery in each tissue, the input to tunits was the output of dREG and bigWig files that were merged across all replicates and crosses. Scripts can be found here: https://github.com/Danko-

Lab/F1 8Organs/blob/main/Tunit predict manuscript.sh https://github.com/Danko-Lab/F1 80rgans/blob/main/run.hmm.h5 F1bedgraph.R https://github.com/Danko-Lab/F1 80rgans/blob/main/hmm.prototypes.R

Clustering: We used all transcripts that are 10,000 bp long from GENCODE vM25. Only reads mapped to the gene body (500bp downstream of the start of the annotation to the end of the annotation) were used. We filtered the transcripts and only kept those with at least 5 mapped reads in every sample. We export rpkm normalized expression estimates of each transcript. Morpheus was used to calculate and plot Spearman's rank correlation 
(https://software.broadinstitute.org/morpheus) with the following parameters: Metric = Spearman rank correlation, Linkage method $=$ Average Linkage, distance. function. name $=$ Spearman rank correlation. Scripts can be found here:

https://github.com/Danko-Lab/F1 80rgans/blob/main/getCounts skipfirst500.R

AlleleHMM: Maternal- and paternal- specific reads mapped using AlleleDB were used as input to AlleleHMM (Chou and Danko, 2019). We combined biological replicates from the same organ and cross, and used the allele-specific read counts as input to AlleleHMM. AlleleHMM blocks were compared with GENOCODE gene annotations to pick the free parameter, $\mathrm{T}$, which maximized sensitivity and specificity for computing entire gene annotations, as described (Chou and Danko, 2019). Most organs used a t of either 1E-5 (brain, liver, spleen, skeletal muscle) or 1E-4 (heart, large intestine, kidney, and stomach). As reported, the primary parameter that influenced $\mathrm{t}$ was the library sequencing depth. AlleleHMM scripts can be found here:

https://github.com/Danko-Lab/AlleleHMM https://github.com/Danko-Lab/F1 80rgans/blob/main/01 F1Ts AlleleHMM.bsh

Discovering strain effect and imprinted domains: We used the following rules to merge nearby allele specific transcription events into starin effect or imprinted domains:

1. Identify candidate AlleleHMM blocks using pooled ChRO-seq reads from samples with the same organ and same cross direction.

2. Combine blocks above from the same organ (but different crosses). Combine $p$-values using Fisher's method for all biological replicates within the same tissue and cross direction. Keep blocks that are biased in the same direction with a Fisher's $p$-value $<=$ 0.05 .

3. Determine whether the blocks are under a strain effect (allelic biased to the same strain in reciprocal crosses) or parent-of-origin imprinted effect (allelic biased to the same parent in reciprocal crosses).

4. Merge overlapping strain effect blocks from different organs into strain effect domains; merge overlapping strain effects from the same imprinted blocks into imprinted domains.

Scripts implementing these rules can be found here: https://github.com/Danko-

Lab/F1 8Organs/blob/main/Find consistent blocks v3.bsh

After discovering blocks, we examined the number of gene annotations in each domain (Fig. 1E). We used GENCODE annotated genes (vM25). We kept all gene annotations and merged those which overlapped or bookended (directly adjacent to, as defined by bedTools) on the same strand so that they were counted once. All operations were performed using bedTools (Quinlan and Hall, 2010). Scripts can be found here:

https://github.com/Danko-Lab/F1 80rgans/blob/main/Find consistent blocks v3.bsh https://github.com/Danko-Lab/F1 80rgans/blob/main/Imprinted figures.R 
Determining the allelic bias state of annotated genes: We used GENCODE gene annotations representing protein-coding genes (VM20) in which the transcription start site overlapped a site identified using dREG (Wang et al., 2018). We used de novo annotations by the tunits package to identify unannotated transcription units, which do not overlap an annotated, active gene as a source of candidate transcribed non-coding RNAs. Transcription units from both sources were merged for downstream analysis. We determine if the gene/ncRNA are allelic biased by comparing mapped reads to the B6 and CAST genomes using a binomial test, retaining transcription units with a $10 \%$ false discovery rate (FDR). Allele specific transcription units were classified as being under a strain effect (allelic biased to the same strain in reciprocal crosses) or parent-of-origin imprinted effect (allelic biased to the same parent in reciprocal crosses).

Scripts can be found: https://github.com/Danko-

Lab/F1 80rgans/blob/main/Genetics or imprinting v2.bsh

Evaluate the contribution of false negatives to organ-specific allelic bias in organ-specific allelic biased domains (OSAB domain): In Supp Fig. 1A and B, we asked whether organs in which we did not identify allelic bias were false negatives. To do this we compared distributions of the transcription level in putatively unbiased organs. For each OSAB domain identified in at least one, but not in all organs, we examined the effect size of allelic bias in the organ with the highest expression that is putatively unbiased. We defined the effect size of allelic bias as the ratio between maternal and paternal reads in the candidate OSAB domain. If the allelic-biased organ was maternally biased, the effect size was calculated as maternal reads divided by paternal reads in the blocks, otherwise the effect size was calculated as paternal reads divided by maternal reads in the blocks. Scripts implementing this can be found here:

https://github.com/Danko-Lab/F1 80rgans/blob/main/AllelicBiase expressionLevel.bsh https://github.com/Danko-

Lab/F1 8Organs/blob/main/getNonBiasedHighest Biased AllelicBiaseDistribution.R

Evaluate the contribution of expression to organ-specific allelic biased domains (OSAB domain): In Supp Fig. 1C, we asked whether OSAB domains were not actively transcribed in candidate unbiased organs. Using bedtools and in-house scripts, we calculated the rpkm (Reads per kilobase per million mapped reads) normalized transcription level of each strain effect block located within the OSAB domains in each organ. The full diploid genome was used for mapping. The non-allelic-biased organs with highest rpkm (nonBiasedH) were selected to compare with the rpkm of the allelic-biased organs in OSAB domains. Scripts implementing this can be found here:

https://github.com/Danko-Lab/F1 80rgans/blob/main/AllelicBiase expressionLevel.bsh https://github.com/DankoLab/F1 8Organs/blob/main/getNonBiasedHighest Biased TotalReadCountRatio.R

\section{Analysis of allele-specific initiation:}

Identification of candidate transcription initiation sites: We used 5 prime end of ChRO-seq reads (the R1 paired-end sequencing file, which represents the 5 prime end of the nascent RNA) to identify candidate initiation sites using methods adapted from (Tome et al., 2018). Briefly, 
candidate transcription start nucleotides (TSN) from each read were merged into candidate transcription start sites, in which the max TSN was identified. We identified candidate TSNs that fall within dREG sites and were supported by at least 5 separate reads. TSNs within $60 \mathrm{bp}$ of each other were merged into candidate TSSs. The TSN with the maximal read depth in each TSS was defined as the maxTSN for that TSS. We allow each TSS to have more than one maxTSNs if multiple TSNs share the same number of read counts in that TSS. To test whether the candidate maxTSNs represented bona-fide transcription start sites, we generated sequence logos centered on the maxTSN using the seqLogo R package (Bembom, 2019). We retained tissues in which the maxTSN contained a clearly defined initiator dinucleotide that reflects a similar sequence composition as those previously reported (Tome et al., 2018). Additionally, we used an in-house $\mathrm{R}$ script to examine the relationship between TSN counts and Read counts of the TSS (Supp Fig2 B), and found a similar relationship to those reported (Tome et al., 2018).

Identify allele specific differences in TSSs abundance (ASTSS abundance): We used a binomial test to identify candidate allele specific transcription start sites, with an expected allelic ratio of 0.5. We filtered candidate allele specific differences using a false discovery rate (FDR) corrected $\mathrm{p}$-value of 0.1 , corresponding to an expected $10 \%$ FDR.

Identify allele specific differences in TSS shape (ASTSS shape): We used a KolmogorovSmirnov (K-S) test to identify TSSs where the distribution of transcription initiation differed significantly between the B6 and CAST alleles (ASTSS shape). We used TSS sites with at least 5 mapped reads specific to the B6 genome and at least 5 mapped reads specific to CAST. Only autosomes were used. We corrected for multiple hypothesis testing using the false discovery rate (Storey and Tibshirani, 2003) and filtered ASTSS shapes using a 10\% FDR. We further separated the ASTSS shape candidates into two groups: one driven by a single base (single base driven ASTSS shape), the other reflecting changes in more than one base in the TSS (multiple base driven ASTSS shape). To separate into two groups, we masked the TSN with the highest allelic difference (determined by read counts) within each TSS and performed a second K-S test. Multiple base driven ASTSS were defined as those which remained significantly different by K-S test after masking the position of highest allelic difference. Single base driven ASTSSs were defined as ASTSSs that were no longer significantly different by K-S test after masking the maximal position. In the second K-S test, we used the nominal p-value defined as the highest nominal p-value that achieved a 10\% FDR during the first K-S test.

SNP analysis: We examined the distribution of single nucleotide polymorphisms (SNPs) near ASTSSs from each class. A major confounding factor in SNP distribution is the ascertainment bias of requiring at least one tagged SNP to define the allelic imbalance between the two alleles, resulting in an enrichment of SNPs within the read. To control for this bias, we compared the set of sites with a significant change in the TSS shape or abundance (FDR $<=0.1$ ) with a background control set defined as candidate TSSs in which there was no evidence of change between alleles (FDR > 0.9) in all analyses. We display a bin size of $5 \mathrm{bp}$. To test for differences, we merged adjacent bins by using a bin size of $10 \mathrm{bp}$ to increase statistical power and tested for enrichment using Fisher's exact test, FDR cutoff $=0.05$. (Fig. 3E,F). We also examined the difference in base composition between the allele with high and low initiation in 
each ASTSS shape difference centered on the position of the maxTSN in the allele with high initiation (in Fig. 3G). We determined the high/low allele based on the transcription level at maxTSN. If there are more than one TSNs with the max read counts, there will be more than one maxTSNs representing each TSS.

Comparison of AT consent between alleles: As a proxy for melting temperature (in Fig. $3 \mathrm{H}$ ), we examined the AT content in 5 bp windows around the maxTSN on alleles with high and low maxTSN usage. As in the SNP analysis (above), we compared the set of sites with a significant change in the TSS shape or abundance (FDR $<=0.1$ ) with a background control set defined as candidate TSSs in which there was no evidence of change between alleles (FDR > 0.9). Computations were performed using $\mathrm{R}$ library TmCalculator (Li, 2019). We used Fisher's exact test to examine if there was an enrichment of AT(in the high allele) to GC (in the low allele) SNPs in each 5 bp bin, and adopted an FDR corrected $p$-value cutoff $=0.05$. In all analyses, positions at -1 and 0 relative to the maxTSN were masked to avoid confounding effects of the initiator sequence motif on computed AT content.

Shooting gallery: In our analysis of the shooting glaray model, we focused on a subset of TSSs which do not appear to change expression globally, and which have a SNP in the initiator element. Toward this end, we identified TSSs which do not overlap AlleleHMM blocks. We set the allele with high and low expression based on allele specific reads in the maxTSN. Next, we divided data into a test and background control dataset in which the test set had a CA dinucleotide in the allele with high maxTSN use and any other combination except for CA on the other allele. The control set did not have a CA dinucleotide in the maxTSN initiator position. Next we computed the distance to the maxTSN and the allelic read count at other candidate initiator motifs (including CA, CG, TA, TG). In all analyses, we compared the set of maxTSNs with SNPs in the initiator position with the control set which did not have a SNP. Statistical tests used an unpaired Wilcoxon rank sum test. We corrected for multiple hypothesis testing using false discovery rate.

All scripts implementing analysis of allele-specific initiation can be found at: https://github.com/Danko-Lab/F1 80rgans/tree/main/initiation

\section{Analysis of allele-specific pause:}

Identification of allele specific differences in pause site shape: All pause analysis focused on ChRO-seq data in three organs (heart, skeletal muscle, and kidney) which used a single base run-on of all four biotin nucleotides. We first focused our analysis on dREG sites in each tissue to identify regions enriched for transcription start and pause sites. We retained dREG sites in which we identified at least 5 reads mapping from both B6 and Cast alleles. We performed a K$S$ test to identify all candidate dREG sites that contained a candidate difference in pause, filtering for a false discovery rate of $0.1(n=2784)$. To examine the relationship between initiation and pause, we identified the maxTSN and maxPause on the B6 and Cast allele separately using reads tagged with a SNP or indel. Since maxTSN and maxPause were defined independently, the maxPause was not always correctly paired with the maxTSN (Tome et al., 
2018). We therefore used $2260 \mathrm{dREG}$ sites where allelic maxPause were 10 to $50 \mathrm{bp}$ downstream of allelic maxTSN on both alleles. These analyses pertain to Fig. $5 \mathrm{~A}$ and $\mathrm{B}$.

Identify genetic determinants of pausing: To focus on the genetic determinants of pausing that were independent of initiation, we identified changes in which the same maxTSN had different allelic maximal pause sites between the Cast and B6 alleles as follows. We used a K-S test to identify maxTSNs with a difference in the maxPause site between alles, filtering for maxTSNs with a different maxPause between alleles and a 10\% FDR in a K-S test $(n=269)$. In most analyses, we also draw a background set in which there was no evidence that sites sharing the same maxTSN had different maxPause sites between alleles, by identifying maxTSNs that have the same maxPause position and a K-S test FDR $>0.9(n=1396)$. In all analyses, we also filtered for maxTSNs with at least 5 allelic reads and B6/CAST read ratio between 0.5 and 2 .

Comparing GC content near the maxPause position: We compared the $\mathrm{G}, \mathrm{C}$, and $\mathrm{GC}$ content between alleles. We computed the $\mathrm{G}, \mathrm{C}$, and $\mathrm{GC}$ content as a function of position relative to maxPause. All of the G, C, and GC contents were combined across unique pause sites from all three organs for which we had single base resolution data $(n=3456)$. We compared three blocks: block 1 was 11 to $20 \mathrm{nt}$ upstream of maxPause, block 2 was 1 to $10 \mathrm{nt}$ upstream of maxPause, and block 3 was 1 to 10 nt downstream of maxPause.

All scripts implementing analysis of allele-specific pause can be found at:

https://github.com/Danko-Lab/F1 80rgans/tree/main/pause

\section{Analysis of allele-specific termination:}

Definition of allelic differences in termination: We noticed frequent AlleleHMM blocks near the 3' end of annotated genes. We used the transcription units (tunits) predictions which overlapped annotated protein coding genes (vM25), as these generally retained the window between the polyadenylation cleavage site and the transcription termination site. We identified transcription units that have AlleleHMM blocks starting within the transcription unit and that end in the final $10 \%$ of the transcription unit or after the transcription unit. The overlapping region between the tunits and AlleleHMM blocks were called candidate allelic termination (AT) windows. To avoid obtaining candidate AT windows that reflected entire transcription units, we retain only AT windows whose length was less than or equal to $50 \%$ length of the host transcription unit.

RNA-seq analysis: Allele specific mapped RNA-seq reads using STAR (see above) were used as input to AlleleHMM to identify the region showing candidate allelic difference in mature mRNA. The transcription units that contain allelic termination windows, as defined above, were separated into two groups: One has an allelic difference in mature mRNA and the other does not. Those with an allelic difference in mature mRNA were defined as having the RNA-seq AlleleHMM blocks between $10 \mathrm{~Kb}$ upstream of the AT windows to the end of the AT windows.

RNA stability analysis: We asked whether there was an allelic difference in mRNA stability between transcription units in which the allelic differences in termination affects the mature 
mRNA and those in which it does not. The RNA stability was defined as in (Blumberg et al., 2021). The stability was defined as the ratio of RNA-seq read counts in exons to ChRO-seq read counts across the gene body. We used gene annotations from GENCODE (vM25). The RNA-seq reads were counted strand specifically using htseq-count. ChRO-seq reads were counted in a strand-specific fashion using in-house $R$ scripts. After removing the genes with less than 10 B6-specific ChRO-seq and less than 10 CAST-specific ChRO-seq reads, the cumulative distribution functions were drawn. All differences were compared using a one-sided K-S test to compare differences in allelic RNA stability between groups.

All scripts implementing analysis of allele-specific termination can be found at: https://github.com/Danko-Lab/F1 80rgans/tree/main/termination

\section{Acknowledgements}

We thank Maria Garcia-Garcia, Abdullah Ozer, John Lis, Hojoong Kwak, Gilad Barshad, Alexandra Chivu, and all members of the Danko lab for valuable discussions and suggestions throughout the life of this project. We thank Peter Borst for help preparing and working with F1 hybrid mice and Jen Grenier and the Cornell TREx facility for preparing mRNA-seq libraries. We thank C. Kaplan (U. Pittsburgh) for rapid constructive comments based on our bioRxiv preprint. Work in this publication was supported by R01-HG010346 and R01-HG009309 (NHGRI) to CGD. The content is solely the responsibility of the authors and does not necessarily represent the official views of the US National Institutes of Health. Some of the figures in this manuscript were created using BioRender. All data are available at Gene Expression Omnibus under the accession number GSE174171.

\section{Figure Captions:}

Figure 1: Reciprocal hybrid cross to understand the Pol II transcription cycle.

(A) Cartoon illustrates the reciprocal F1 hybrids cross design between the strains C57BL/6J (B6) and CAST/EiJ (CAST). We have seven independent crosses (3x C57BL/6 x Cast and 4x Cast x C57BL/6).

(B) Spearman's rank correlation of ChRO-seq signals in gene bodies. The color on the top indicates the direction of crosses: Black is B6 $\times$ CAST, brown is CAST $\times$ B6. The cartoon on the right indicates the organ each sample was harvested from.

(C) Cartoon depicts the methods used to identify allelic biased blocks, domains, and how they were classified as a strain-associated effect or imprinted.

(D) The pie chart shows the proportion of domains under strain effect or imprinted.

(E) The histogram shows the proportion of domains as a function of the number of gencode gene annotations in each domain.

(F) The browsershot shows an example of ChRO-seq data that has an imprinted domain (top row). The second and third rows show the imprinted protein-coding genes and imprinted non-coding RNA (ncRNA) from all organs. (BN:brain, LV:liver, SK:skeletal 
muscle, Gl: large intestine, HT: heart, KD: kidney, P: paternal, M: maternal). The yellow shade indicates the imprinted regions in the brain and liver.

(G) The histogram shows the proportion of domains as a function of the domain length.

(H) The bar chart shows the percentage of domains as a function of the number of organs with allelic biased blocks in the domain.

Supplemental Figure 1:

(A) The histogram shows the frequency of blocks within organ-specific allelic biased domains (OSAB domain) as a function of effect size. Red (Biased) is from the organ with OSAB domain. Blue (NonBiased) is from the organ with the highest expression that is putatively unbiased. If the allelic-biased organ was maternally biased, the effect size was calculated as maternal reads divided by paternal reads in the blocks, otherwise the effect size was calculated as paternal reads divided by maternal reads in the blocks.

(B) The histogram shows the frequency of blocks within the OSAB domain as a function of maternal reads ratio. Red (Biased) is from the organ with OSAB domain. Blue (NonBiased) is from the organ with the highest expression that is putatively unbiased.

(C) The histogram shows the frequency of blocks within OSAB domain as a function of the log2 ratio between the rpkm of the non-allelic-biased organs with highest rpkm (nonBiasedHighest) and the allelic-biased organs in OSAB domains.

Figure 2: Features associated with imprinting domains.

(A) The bar charts show the number of imprinted (top) and strain effect domains (bottom) in each organ. (BN:brain, SP: spleen, HT: heart, SK:skeletal muscle, KD: kidney, ST:stomach, Gl: large intestine, LV:liver)

(B) The browsershot shows allele-specific ChRO-seq signal from the lgf2r locus in brain, which contains the imprinting-associated ncRNA, Airn. M: maternal-specific reads; P: paternal-specific reads.

(C) Stacked bar charts show the percentage of strain effect and imprinted domains that contain transcription units with opposite allelic bias (called switches). Those without allelic bias switches were consistently biased to the same direction (for imprinted domain, all maternally-biased or all paternally-biased, no switch). Those domains with allelic bias switches contain blocks biased to different directions (some maternallybiased and some paternally-biased, with switches).

Figure 3: Allele specific effects on the distribution of transcription initiation.

(A) The ChRO-seq signals of the 5' end of the nascent RNA were used to define transcription start nucleotides (TSNs), or the individual bases with evidence of transcription initiation. TSNs within $60 \mathrm{bp}$ were grouped into transcription start sites (TSSs).

(B) The browsershot shows an example of allelic differences in the shape of TSS that are predominantly explained by a single TSN position (arrowhead).

(C) The browsershot shows an example of allelic differences in TSS driven by multiple TSNs within the same TSS, arrowheads indicate several prominent positions with allelic differences in Poll abundance. 
(D) Stacked bar chart shows the average proportion of allelic differences in TSSs driven by a single TSN (red). The specific proportion for each of the six organs are shown by dots. Six organs were selected that showed a strong signal of Inr motif at the maxTSNs (brain, heart, liver, large intestine, stomach, and spleen).

(E) Scatterplot shows the average SNP counts as a function of distance to the maxTSN at sites showing allelic differences in TSSs driven by a single base in the liver. Red denotes changes in TSS shape (Kolmogorov-Smirnov (KS) test; FDR $<=0.10$ ); black indicates TSSs without evidence for differences in TSS shape (KS test; FDR >0.90). Dots represent non-overlapping 5 bp bins. Yellow shade indicates statistically significant differences (false discovery rate corrected Fisher's exact on 10 bp bin sizes, FDR <= $0.05)$

(F) Scatterplot shows the average SNP counts as a function of distance to the maxTSN at sites showing allelic differences in TSS driven by multiple bases in the liver sample. Blue denotes changes in TSS shape classified as multiple TSN driven (Kolmogorov-Smirnov (KS) test; FDR <=0.10); black indicates TSSs without evidence for differences in TSS shape (KS test; FDR > 0.90). Dots represent non-overlapping 5 bp bins. Yellow shade indicates statistically significant differences (false discovery rate corrected Fisher's exact on 10 bp bin sizes, FDR <=0.05)

(G) The scatterplot shows the average difference in base composition between the allele with high and low TSN use around the maxTSN in single-base driven allele specific TSSs. The sequence logo on the bottom represents the high allele in single-base driven allele specific TSSs. The high/low allele were determined by the read depth at maxTSN.

(H) The scatter plots shows the difference of AT contents between the high and low alleles with the maxTSN and -1 base upstream maxTSN masked. Dots represent 5 bp nonoverlapping windows. Red denotes single base driven allele specific TSSs; black denotes control TSSs with no evidence of allele specific changes. The yellow shade indicates a significant enrichment of AT(at high allele) to GC (at low allele) SNPs at each bin (size=5bp; Fisher's exact test, FDR <=0.05).

Supplemental Figure 2:

(A) Sequence logos show the information content around the maxTSNs of each organ.

(B) Scatter plot shows the number of TSNs in a TSS as a function of the read counts in the TSS.

(C) Scatterplot shows the average SNP counts as a function of distance to the maxTSN at sites showing allelic differences in TSSs driven by a single base in the brain. Red denotes changes in TSS shape (Kolmogorov-Smirnov (KS) test; FDR $<=0.10$ ); black indicates TSSs without evidence for differences in TSS shape (KS test; FDR > 0.90). Dots represent non-overlapping 5 bp bins. Yellow shade indicates statistically significant differences (false discovery rate corrected Fisher's exact on 10 bp bin sizes, FDR <= 0.05)

(D) Scatterplot shows the average SNP counts as a function of distance to the maxTSN at sites showing allelic differences in TSS driven by multiple bases in the brain sample. Blue denotes changes in TSS shape classified as multiple TSN driven (FDR corrected Kolmogorov-Smirnov (KS) test; FDR $<=0.10$ ); black indicates TSSs without evidence for 
differences in TSS shape (KS test; FDR > 0.90). Dots represent non-overlapping 5 bp bins. Yellow shade indicates statistically significant differences (false discovery rate corrected Fisher's exact on 10 bp bin sizes, FDR $<=0.05$ )

(E) The scatterplot shows the average difference in base composition between the allele with high and low TSN use around the maxTSN in single-base driven allele specific TSSs. The sequence logo on the bottom represents the high allele in single-base driven allele specific TSSs. The high/low allele were determined by the read depth at maxTSN. This figure denotes TSSs in the brain.

(F) The scatter plot shows the difference of AT contents between the high and low alleles in the brain with the maxTSN and -1 base upstream maxTSN masked. Dots represent $5 \mathrm{bp}$ non-overlapping windows. Red denotes single base driven allele specific TSSs; black denotes control TSSs with no evidence of allele specific changes. The yellow shade indicates a significant enrichment of AT(at high allele) to GC (at low allele) SNPs at each bin (size=5bp; Fisher's exact test, FDR <=0.05).

(G) The scatter plots show the difference of AT contents between the high and low alleles in liver with the maxTSN and -1 base upstream maxTSN masked. This plot shows the multiple base driven allele specific TSS in the liver samples. Dots represent 5 bp nonoverlapping windows. Blue denotes multiple base driven allele specific TSSs; black denotes control TSSs with no evidence of allele specific changes. The yellow shade indicates a significant enrichment of AT(at high allele) to GC (at low allele) SNPs at each bin (size=5bp; Fisher's exact test, FDR $<=0.05$ ).

(H) The scatter plots show the difference of AT contents between the high and low alleles with the maxTSN and -1 base upstream maxTSN masked. This plot shows the multiple base driven allele specific TSS in the brain samples. Dots represent 5 bp nonoverlapping windows. Blue denotes multiple base driven allele specific TSSs; black denotes control TSSs with no evidence of allele specific changes. The yellow shade indicates a significant enrichment of AT(at high allele) to GC (at low allele) SNPs at each bin (size=5bp; Fisher's exact test, FDR <=0.05).

Figure 4: Browian motion model of transcription start nucleotide selection.

(A) Cartoon shows our expectation of the effects of allelic DNA sequence variation on transcription start nucleotide selection based on the "shooting gallery" model, in which Pol II initiates at potential TSNs (triangles) after DNA melting. We expected that mutations in a strong initiator dinucleotide (CA) on (for example) the CAST allele (bottom) would shift initiation to the initiator elements further downstream. The size of the triangle indicates the strength of the initiator.

(B) The violin plots show the distribution of ChRO-seq signal ratios on the candidate initiator motifs (including CA, CG, TA, TG) within $20 \mathrm{bp}$ of the maxTSNs that had a CA dinucleotide in the allele with high maxTSN (SNP in Inr, purple) or had a CA dinucleotide in both alleles (No SNP in Inr, gray). Note that the central maxTSN was not included in the analysis. Wilcoxon rank sum test with continuity correction is $p$-value $=5.665 \mathrm{e}-10$ for Brain and $p$-value $<2.2 e-16$ for liver.

(C) The box plots show the distribution of ChRO-seq signals ratios at TSNs with any variation of the initiator motif (including CA, CG, TA, TG) as a function of the distance 
from the maxTSNs that had a CA dinucleotide in the allele with high maxTSN (SNP in Inr, purple) or had a CA dinucleotide in both alleles (No SNP in Inr, gray). Yellow shade indicates Wilcoxon Rank Sum and Signed Rank Tests (SNP in Inr vs no SNP in Inr) with $\mathrm{fdr}<=0.05$. The TSSs were combined from the brain and liver samples.

(D) The browser shot shows an example of a maxTSN with increased initiation upstream and downstream of an allelic change in a CA dinucleotide. The orange block AG denotes a SNP at the maxTSN, in which B6 contains the high maxTSN with CA and CAST contains CG. The ChRO-seq signals at the alternative TSN with a CA dinucleotide (arrow head) upstream of the maxTSN were higher in the low allele (CAST in this case), resulting in a different maxTSN in CAST.

Supplemental Figure 3:

Violin plots show the distribution of $\mathrm{ChRO}$-seq signals ratios between high low alleles at the candidate initiator motifs (All Inr : CA, CG, TA, TG; OnlyWeak Inr : CG, TA, TG; and OnlyCA) that are within 20bp of the maxTSNs that had a CA dinucleotide in the allele with high maxTSN (SNP in Inr, purple) or had a CA dinucleotide in both alleles (No SNP in Inr, gray) in Brain(BN) or Liver(LV). Single: indicates Single base driven allele-specific TSSs. Multiple: indicates Multiple base driven allele-specific TSSs. Yellow shade indicates Wilcoxon Rank Sum and Signed Rank Tests (SNP in Inr vs no SNP in Inr) with $\mathrm{fdr}<=0.05$.

Figure 5: Allele specific effects on the distribution of Pol II in the promoter proximal pause.

(A) Scatterplots show the relationship between distances of allelic maxPause and allelic maxTSN within dREG sites with allelic different pause $(n=2,260)$.

(B) Top histogram shows the number of sites as a function of the distance between allelic maxTSN in which the allelic maxPause was identical $(n=359)$. Bottom histogram shows the number of sites as a function of the distance between allelic maxPause where the allelic maxTSN was identical $(n=823)$.

(C) Scatterplot shows the relationship between indel length and the allelic difference of the average pause position on the reference genome (mm10). The pause positions of CAST were first determined in the CAST genome and then liftovered to mm10. Only sites initiated from the maxTSN and with allelic difference in pause shape were shown (KS test, $\mathrm{fdr}<=0.1$; also requiring a distinct allelic maximal pause). Color indicates the organs from which the TSN-pause relationship was obtained.

(D) Top: scatterplot shows the average SNPs per base around the position of the Pol II in which the distance between the maxTSN and the max pause was lowest (short pause). Red represents sites with allelic difference in pause shape (Allelic pause difference, KS test, fdr $<=0.1$ with distinct allelic maxPause, $n=269$ ), Blue is the control group (No allelic pause difference, $\mathrm{KS}$ test, $\mathrm{fdr}>0.9$ and the allelic maxPause were identical, $\mathrm{n}=$ 1,396). Bottom: The sequence logo obtained from the maxPause position based on all reads ( $n=3,456$ max pause sites). Sites were combined from three organs, after removing pause sites that were identical between organs. 
(E) Violin plots show the G content, GC content and C content as a function of position relative to maxPause defined using all reads (combined from three organs with duplicate pause sites removed, $n=3,456$ ), block 1 was 11 to 20 nt upstream of maxPause, block 2 was 1 to $10 \mathrm{nt}$ upstream of maxPause, and block 3 was 1 to $10 \mathrm{nt}$ downstream of maxPause $\left({ }^{*} p<0.05,{ }^{* *} p<0.01,{ }^{* * *} p<0.001,{ }^{* * *} p<0.0001\right)$. Block 2 had a higher $\mathrm{G}$ content and a lower $\mathrm{C}$ content than the two surrounding blocks.

(F) Pie charts show the proportion of different events around the maxPause of short alleles (short pause) with or without allelic pause differences. Sites were combined from three organs with duplicated pause sites removed.

(G) Sequence logos show the sequence content of short alleles and long alleles at 270 short pause sites and 278 long pause sites.

$(\mathrm{H})$ The histograms show the fraction of pause sites as a function of distance between allelic maxPause, i.e. the distance between the short and long pause. The lines show the cumulative density function. Blue represents pause sites with allelic differences $(\mathrm{n}=$ $285)$; red is a subgroup of blue sites with a $C$ to $A / T / G$ SNP at the maxpause $(n=34)$. Two-sample Kolmogorov-Smirnov test $p$-value $=0.002694$

(I) The histograms show the fraction of pause sites as a function of distance between allelic maxPause. The lines show the cumulative density function. Blue is pause sites with allelic differences $(n=285)$, green is a subgroup of blue sites that contain indels between initiation and long pause sites $(n=60)$, Two-sample Kolmogorov-Smirnov test $p$ value $=0.02755$

Supplemental Figure 4:

Spearman's rank correlation of the ChRO-seq data, including samples with a single base resolution for the Pol II active site. Samples with single nucleotide precision are shown on the top in red bold font and at the right with star. (BN:brain, GI: large intestine, ST: Stomach, LV:liver, KD: kidney, SP:spleen, HT: heart, SK:skeletal muscle, MB6: B6 x CAST, PB6: CAST x B6)

Supplemental Figure 5:

(A) Sequence logos show the information contents around the initiation site (see Methods).

(B) Scatter plots show the difference of nucleotide usage between short and long alleles as a function of distance to allelic maxPause.

Figure 6: Widespread allele specific differences in the Pol II termination site.

(A) The browser shot shows an example of allelic termination differences (yellow shade) in both brain and liver. Pol II terminates earlier on the B6 allele, resulting in a longer transcription unit on the CAST allele. The difference in allelic read abundance was identified by AlleleHMM. We defined the allelic termination difference (yellow shade) using the intersection between the transcription unit and AlleleHMM blocks.

(B) Heatmaps show the raw read counts in transcription units (blue bar) with an allelic termination difference (yellow bar), centered at the beginning of allelic termination (solid triangle). The heatmap bin size is $500 \mathrm{bp}$, and $20 \mathrm{~kb}$ is shown upstream and downstream. The rows were sorted by the length of allelic termination differences 
determined by ChROseq signals from Liver. The short and long alleles were determined based on analysis of the liver.

(C) The histogram shows the fraction of transcription units as a function of the length of allelic termination difference .

(D) Pie charts show the proportion of transcription units with allelic termination difference that also contains allelic difference in mature mRNA (orange).

Supplemental Figure 6:

(A) Scatterplots show the relationship between the ChRO-seq signal in the transcription unit (defined using the tunits program; see Methods) and the region showing an allelic termination difference.

(B) Violin plots show the distribution of the length of allelic termination in eight organs.

(C) Scatterplots represent the cumulative density function of allelic RNA stability differences in brain samples. Two-sample Kolmogorov-Smirnov tests, $p$-value $=0.9995$.

(D) The lines show the cumulative density function of the allelic RNA stability difference in the liver samples. Two-sample Kolmogorov-Smirnov tests, $p$-value $=0.7542$.

Supplemental Figure 7: Nucleic acid sequences used in custom barcoded high throughput adapters.

Supplemental Figure 8: Figure depicts the nucleic acid sequences used in the small RNA designed adapters.

\section{References}

Andergassen D, Dotter CP, Wenzel D, Sigl V, Bammer PC, Muckenhuber M, Mayer D, Kulinski TM, TheussI H-C, Penninger JM, Bock C, Barlow DP, Pauler FM, Hudson QJ. 2017. Mapping the mouse Allelome reveals tissue-specific regulation of allelic expression. Elife 6. doi:10.7554/eLife.25125

Augui S, Nora EP, Heard E. 2011. Regulation of X-chromosome inactivation by the Xinactivation centre. Nat Rev Genet 12:429-442.

Battle A, Mostafavi S, Zhu X, Potash JB, Weissman MM, McCormick C, Haudenschild CD, Beckman KB, Shi J, Mei R, Urban AE, Montgomery SB, Levinson DF, Koller D. 2014.

Characterizing the genetic basis of transcriptome diversity through RNA-sequencing of 922 individuals. Genome Res 24:14-24.

Bembom O. 2019. seqLogo: Sequence logos for DNA sequence alignments.

Blumberg A, Zhao Y, Huang Y-F, Dukler N, Rice EJ, Chivu AG, Krumholz K, Danko CG, Siepel A. 2021. Characterizing RNA stability genome-wide through combined analysis of PRO-seq and RNA-seq data. BMC Biol 19:30.

Braberg H, Jin H, Moehle EA, Chan YA, Wang S, Shales M, Benschop JJ, Morris JH, Qiu C, Hu F, Tang LK, Fraser JS, Holstege FCP, Hieter P, Guthrie C, Kaplan CD, Krogan NJ. 2013. From structure to systems: high-resolution, quantitative genetic analysis of RNA polymerase II. Cell 154:775-788.

Breslauer KJ, Frank R, Blöcker H. 1986. Predicting DNA duplex stability from the base sequence. Proceedings of the.

Carninci P, Kasukawa T, Katayama S, Gough J, Frith MC, Maeda N, Oyama R, Ravasi T, 
Lenhard B, Wells C, Kodzius R, Shimokawa K, Bajic VB, Brenner SE, Batalov S, Forrest ARR, Zavolan M, Davis MJ, Wilming LG, Aidinis V, Allen JE, Ambesi-Impiombato A, Apweiler R, Aturaliya RN, Bailey TL, Bansal M, Baxter L, Beisel KW, Bersano T, Bono H, Chalk AM, Chiu KP, Choudhary V, Christoffels A, Clutterbuck DR, Crowe ML, Dalla E, Dalrymple BP, de Bono B, Della Gatta G, di Bernardo D, Down T, Engstrom P, Fagiolini M, Faulkner G, Fletcher CF, Fukushima T, Furuno M, Futaki S, Gariboldi M, Georgii-Hemming P, Gingeras TR, Gojobori T, Green RE, Gustincich S, Harbers M, Hayashi Y, Hensch TK, Hirokawa N, Hill D, Huminiecki L, lacono M, Ikeo K, Iwama A, Ishikawa T, Jakt M, Kanapin A, Katoh M, Kawasawa Y, Kelso J, Kitamura H, Kitano H, Kollias G, Krishnan SPT, Kruger A, Kummerfeld SK, Kurochkin IV, Lareau LF, Lazarevic D, Lipovich L, Liu J, Liuni S, McWilliam S, Madan Babu M, Madera M, Marchionni L, Matsuda H, Matsuzawa S, Miki H, Mignone F, Miyake S, Morris K, Mottagui-Tabar S, Mulder N, Nakano N, Nakauchi H, Ng P, Nilsson R, Nishiguchi S, Nishikawa S, Nori F, Ohara O, Okazaki Y, Orlando V, Pang KC, Pavan WJ, Pavesi G, Pesole G, Petrovsky N, Piazza S, Reed J, Reid JF, Ring BZ, Ringwald M, Rost B, Ruan Y, Salzberg SL, Sandelin A, Schneider C, Schönbach C, Sekiguchi K, Semple CAM, Seno S, Sessa L, Sheng Y, Shibata Y, Shimada H, Shimada K, Silva D, Sinclair B, Sperling S, Stupka E, Sugiura K, Sultana R, Takenaka Y, Taki K, Tammoja K, Tan SL, Tang S, Taylor MS, Tegner J, Teichmann SA, Ueda HR, van Nimwegen E, Verardo R, Wei CL, Yagi K, Yamanishi H, Zabarovsky E, Zhu S, Zimmer A, Hide W, Bult C, Grimmond SM, Teasdale RD, Liu ET, Brusic V, Quackenbush J, Wahlestedt C, Mattick JS, Hume DA, Kai C, Sasaki D, Tomaru Y, Fukuda S, KanamoriKatayama M, Suzuki M, Aoki J, Arakawa T, lida J, Imamura K, Itoh M, Kato T, Kawaji H, Kawagashira N, Kawashima T, Kojima M, Kondo S, Konno H, Nakano K, Ninomiya N, Nishio T, Okada M, Plessy C, Shibata K, Shiraki T, Suzuki S, Tagami M, Waki K, Watahiki A, Okamura-Oho Y, Suzuki H, Kawai J, Hayashizaki Y, FANTOM Consortium, RIKEN Genome Exploration Research Group and Genome Science Group (Genome Network Project Core Group). 2005. The transcriptional landscape of the mammalian genome. Science 309:1559-1563.

Carninci P, Sandelin A, Lenhard B, Katayama S, Shimokawa K, Ponjavic J, Semple CAM, Taylor MS, Engström PG, Frith MC, Forrest ARR, Alkema WB, Tan SL, Plessy C, Kodzius R, Ravasi T, Kasukawa T, Fukuda S, Kanamori-Katayama M, Kitazume Y, Kawaji H, Kai C, Nakamura M, Konno H, Nakano K, Mottagui-Tabar S, Arner P, Chesi A, Gustincich S, Persichetti F, Suzuki H, Grimmond SM, Wells CA, Orlando V, Wahlestedt C, Liu ET, Harbers M, Kawai J, Bajic VB, Hume DA, Hayashizaki Y. 2006. Genome-wide analysis of mammalian promoter architecture and evolution. Nat Genet 38:626-635.

Chen J, Rozowsky J, Galeev TR, Harmanci A, Kitchen R, Bedford J, Abyzov A, Kong Y, Regan L, Gerstein M. 2016. A uniform survey of allele-specific binding and expression over 1000Genomes-Project individuals. Nat Commun 7:11101.

Cho H, Kim TK, Mancebo H, Lane WS, Flores O, Reinberg D. 1999. A protein phosphatase functions to recycle RNA polymerase II. Genes Dev 13:1540-1552.

Chou S-P, Danko CG. 2019. AlleleHMM: a data-driven method to identify allele specific differences in distributed functional genomic marks. Nucleic Acids Res. doi:10.1093/nar/gkz176

Chu T, Rice EJ, Booth GT, Salamanca HH, Wang Z, Core LJ, Longo SL, Corona RJ, Chin LS, Lis JT, Kwak H, Danko CG. 2018. Chromatin run-on and sequencing maps the transcriptional regulatory landscape of glioblastoma multiforme. Nat Genet 50:1553-1564.

Danko CG, Choate LA, Marks BA, Rice EJ, Wang Z, Chu T, Martins AL, Dukler N, Coonrod SA, Tait Wojno ED, Lis JT, Kraus WL, Siepel A. 2018. Dynamic evolution of regulatory element ensembles in primate CD4+ T cells. Nature Ecology \& Evolution. doi:10.1038/s41559-0170447-5

Danko CG, Hah N, Luo X, Martins AL, Core L, Lis JT, Siepel A, Kraus WL. 2013. Signaling 
pathways differentially affect RNA polymerase II initiation, pausing, and elongation rate in cells. Mol Cell 50:212-222.

Delaneau O, Zazhytska M, Borel C, Giannuzzi G, Rey G, Howald C, Kumar S, Ongen H, Popadin K, Marbach D, Ambrosini G, Bielser D, Hacker D, Romano L, Ribaux P, Wiederkehr M, Falconnet E, Bucher P, Bergmann S, Antonarakis SE, Reymond A, Dermitzakis ET. 2019. Chromatin three-dimensional interactions mediate genetic effects on gene expression. Science 364 :eaat8266.

Dobin A, Davis CA, Schlesinger F, Drenkow J, Zaleski C, Jha S, Batut P, Chaisson M, Gingeras TR. 2013. STAR: ultrafast universal RNA-seq aligner. Bioinformatics 29:15-21.

Fant CB, Levandowski CB, Gupta K, Maas ZL, Moir J, Rubin JD, Sawyer A, Esbin MN, Rimel JK, Luyties O, Marr MT, Berger I, Dowell RD, Taatjes DJ. 2020. TFIID Enables RNA Polymerase II Promoter-Proximal Pausing. Mol Cell 78:785-793.e8.

Fuda NJ, Ardehali MB, Lis JT. 2009. Defining mechanisms that regulate RNA polymerase II transcription in vivo. Nature 461:186-192.

Gilchrist DA, Dos Santos G, Fargo DC, Xie B, Gao Y, Li L, Adelman K. 2010. Pausing of RNA polymerase II disrupts DNA-specified nucleosome organization to enable precise gene regulation. Cell 143:540-551.

Gressel S, Schwalb B, Cramer P. 2019. The pause-initiation limit restricts transcription activation in human cells. Nat Commun 10:3603.

Gressel S, Schwalb B, Decker TM, Qin W, Leonhardt H, Eick D, Cramer P. 2017. CDK9dependent RNA polymerase II pausing controls transcription initiation. Elife 6. doi:10.7554/eLife.29736

Grünberg S, Warfield L, Hahn S. 2012. Architecture of the RNA polymerase II preinitiation complex and mechanism of ATP-dependent promoter opening. Nat Struct Mol Biol 19:788796.

GTEx Consortium, Laboratory, Data Analysis \&Coordinating Center (LDACC)—Analysis Working Group, Statistical Methods groups-Analysis Working Group, Enhancing GTEx (eGTEx) groups, NIH Common Fund, NIH/NCI, NIH/NHGRI, NIH/NIMH, NIH/NIDA, Biospecimen Collection Source Site-NDRI, Biospecimen Collection Source Site-RPCI, Biospecimen Core Resource-VARI, Brain Bank Repository-University of Miami Brain Endowment Bank, Leidos Biomedical-Project Management, ELSI Study, Genome Browser Data Integration \&Visualization-EBI, Genome Browser Data Integration \&Visualization-UCSC Genomics Institute, University of California Santa Cruz, Lead analysts:, Laboratory, Data Analysis \&Coordinating Center (LDACC):, NIH program management:, Biospecimen collection:, Pathology:, eQTL manuscript working group:, Battle A, Brown CD, Engelhardt BE, Montgomery SB. 2017. Genetic effects on gene expression across human tissues. Nature 550:204-213.

Haberle V, Stark A. 2018. Eukaryotic core promoters and the functional basis of transcription initiation. Nat Rev Mol Cell Biol. doi:10.1038/s41580-018-0028-8

Ho AY, Dimitropoulos A. 2010. Clinical management of behavioral characteristics of Prader-Willi syndrome. Neuropsychiatr Dis Treat 6:107-118.

Jonkers I, Kwak H, Lis JT. 2014. Genome-wide dynamics of Pol II elongation and its interplay with promoter proximal pausing, chromatin, and exons. Elife 3:e02407.

Jonkers I, Lis JT. 2015. Getting up to speed with transcription elongation by RNA polymerase II. Nat Rev Mol Cell Biol 16:167-177.

Kaplan CD, Jin H, Zhang IL, Belyanin A. 2012. Dissection of Pol II trigger loop function and Pol II activity-dependent control of start site selection in vivo. PLoS Genet 8:e1002627.

Kaufmann J, Smale ST. 1994. Direct recognition of initiator elements by a component of the transcription factor IID complex. Genes Dev 8:821-829.

Kristjánsdóttir K, Dziubek A, Kang HM, Kwak H. 2020. Population-scale study of eRNA transcription reveals bipartite functional enhancer architecture. Nat Commun 11:5963. 
Kwak H, Fuda NJ, Core LJ, Lis JT. 2013. Precise maps of RNA polymerase reveal how promoters direct initiation and pausing. Science 339:950-953.

Langmead B, Trapnell C, Pop M, Salzberg SL. 2009. Ultrafast and memory-efficient alignment of short DNA sequences to the human genome. Genome Biol 10:R25.

Lappalainen T, Sammeth M, Friedländer MR, 't Hoen PAC, Monlong J, Rivas MA, GonzàlezPorta M, Kurbatova N, Griebel T, Ferreira PG, Barann M, Wieland T, Greger L, van Iterson M, Almlöf J, Ribeca P, Pulyakhina I, Esser D, Giger T, Tikhonov A, Sultan M, Bertier G, MacArthur DG, Lek M, Lizano E, Buermans HPJ, Padioleau I, Schwarzmayr T, Karlberg O, Ongen H, Kilpinen H, Beltran S, Gut M, Kahlem K, Amstislavskiy V, Stegle O, Pirinen M, Montgomery SB, Donnelly P, McCarthy MI, Flicek P, Strom TM, Geuvadis Consortium, Lehrach H, Schreiber S, Sudbrak R, Carracedo A, Antonarakis SE, Häsler R, Syvänen A-C, van Ommen G-J, Brazma A, Meitinger T, Rosenstiel P, Guigó R, Gut IG, Estivill X, Dermitzakis ET. 2013. Transcriptome and genome sequencing uncovers functional variation in humans. Nature 501:506-511.

Li H, Durbin R. 2009. Fast and accurate short read alignment with Burrows-Wheeler transform. Bioinformatics 25:1754-1760.

Li J. 2019. TmCalculator: Melting Temperature of Nucleic Acid Sequences.

Luse DS, Parida M, Spector BM, Nilson KA, Price DH. 2020. A unified view of the sequence and functional organization of the human RNA polymerase II promoter. Nucleic Acids Res. doi:10.1093/nar/gkaa531

Mahat DB, Kwak H, Booth GT, Jonkers IH, Danko CG, Patel RK, Waters CT, Munson K, Core LJ, Lis JT. 2016. Base-pair-resolution genome-wide mapping of active RNA polymerases using precision nuclear run-on (PRO-seq). Nat Protoc 11:1455-1476.

Mahat DB, Salamanca HH, Duarte FM, Danko CG, Lis JT. 2016. Mammalian Heat Shock Response and Mechanisms Underlying Its Genome-wide Transcriptional Regulation. Mol Cell. doi:10.1016/j.molcel.2016.02.025

Miller T, Krogan NJ, Dover J, Erdjument-Bromage H, Tempst P, Johnston M, Greenblatt JF, Shilatifard A. 2001. COMPASS: A complex of proteins associated with a trithorax-related SET domain protein. Proceedings of the National Academy of Sciences. doi:10.1073/pnas.231473398

Mittleman BE, Pott S, Warland S, Barr K, Cuevas C, Gilad Y. 2021. Divergence in alternative polyadenylation contributes to gene regulatory differences between humans and chimpanzees. Elife 10. doi:10.7554/eLife.62548

Mittleman BE, Pott S, Warland S, Zeng T, Mu Z, Kaur M, Gilad Y, Li Y. 2020. Alternative polyadenylation mediates genetic regulation of gene expression. Elife $\mathbf{9}$. doi:10.7554/eLife.57492

Montgomery SB, Sammeth M, Gutierrez-Arcelus M, Lach RP, Ingle C, Nisbett J, Guigo R, Dermitzakis ET. 2010. Transcriptome genetics using second generation sequencing in a Caucasian population. Nature 464:773-777.

Murakami K, Elmlund H, Kalisman N, Bushnell DA, Adams CM, Azubel M, Elmlund D, LeviKalisman Y, Liu X, Gibbons BJ, Levitt M, Kornberg RD. 2013. Architecture of an RNA Polymerase II Transcription Pre-Initiation Complex. Science 342. doi:10.1126/science.1238724

Muse GW, Gilchrist DA, Nechaev S, Shah R, Parker JS, Grissom SF, Zeitlinger J, Adelman K. 2007. RNA polymerase is poised for activation across the genome. Nat Genet 39:15071511.

Nechaev S, Fargo DC, dos Santos G, Liu L, Gao Y, Adelman K. 2010. Global analysis of short RNAs reveals widespread promoter-proximal stalling and arrest of Pol II in Drosophila. Science 327:335-338.

Orphanides G, LeRoy G, Chang CH, Luse DS, Reinberg D. 1998. FACT, a factor that facilitates transcript elongation through nucleosomes. Cell 92:105-116. 
O'Sullivan JM, Tan-Wong SM, Morillon A, Lee B, Coles J, Mellor J, Proudfoot NJ. 2004. Gene loops juxtapose promoters and terminators in yeast. Nat Genet 36:1014-1018.

Pelc K, Cheron G, Dan B. 2008. Behavior and neuropsychiatric manifestations in Angelman syndrome. Neuropsychiatr Dis Treat 4:577.

Pickrell JK, Marioni JC, Pai AA, Degner JF, Engelhardt BE, Nkadori E, Veyrieras J-B, Stephens M, Gilad Y, Pritchard JK. 2010. Understanding mechanisms underlying human gene expression variation with RNA sequencing. Nature 464:768-772.

Plasschaert RN, Bartolomei MS. 2015. Tissue-specific regulation and function of Grb10 during growth and neuronal commitment. Proc Natl Acad Sci U S A 112:6841-6847.

Qiu C, Jin H, Vvedenskaya I, Llenas JA, Zhao T, Malik I, Visbisky AM, Schwartz SL, Cui P, Čabart P, Han KH, Lai WKM, Metz RP, Johnson CD, Sze S-H, Pugh BF, Nickels BE, Kaplan CD. 2020. Universal promoter scanning by Pol II during transcription initiation in Saccharomyces cerevisiae. Genome Biol 21:132.

Quinlan AR, Hall IM. 2010. BEDTools: a flexible suite of utilities for comparing genomic features. Bioinformatics 26:841-842.

Rahl PB, Lin CY, Seila AC, Flynn RA, McCuine S, Burge CB, Sharp PA, Young RA. 2010. cMyc regulates transcriptional pause release. Cell 141:432-445.

Ranish JA, Yudkovsky N, Hahn S. 1999. Intermediates in formation and activity of the RNA polymerase II preinitiation complex: holoenzyme recruitment and a postrecruitment role for the TATA box and TFIIB. Genes Dev 13:49-63.

Rennie S, Dalby M, van Duin L, Andersson R. 2018. Transcriptional decomposition reveals active chromatin architectures and cell specific regulatory interactions. Nat Commun 9:487.

Rosonina E, Kaneko S, Manley JL. 2006. Terminating the transcript: breaking up is hard to do. Genes Dev 20:1050-1056.

Rougvie AE, Lis JT. 1988. The RNA polymerase II molecule at the 5' end of the uninduced hsp70 gene of D. melanogaster is transcriptionally engaged. Cell 54:795-804.

Rozowsky J, Abyzov A, Wang J, Alves P, Raha D, Harmanci A, Leng J, Bjornson R, Kong Y, Kitabayashi N, Bhardwaj N, Rubin M, Snyder M, Gerstein M. 2011. AlleleSeq: analysis of allele-specific expression and binding in a network framework. Mol Syst Biol 7:522.

Schmieder R, Edwards R. 2011. Quality control and preprocessing of metagenomic datasets. Bioinformatics 27:863-864.

Schwalb B, Michel M, Zacher B, Frühauf K, Demel C, Tresch A, Gagneur J, Cramer P. 2016. TT-seq maps the human transient transcriptome. Science 352:1225-1228.

Sleutels F, Zwart R, Barlow DP. 2002. The non-coding Air RNA is required for silencing autosomal imprinted genes. Nature 415:810-813.

Smale ST, Baltimore D. 1989. The "initiator" as a transcription control element. Cell 57:103-113.

Storey JD, Tibshirani R. 2003. Statistical significance for genomewide studies. Proc Natl Acad Sci U S A 100:9440-9445.

Tome JM, Tippens ND, Lis JT. 2018. Single-molecule nascent RNA sequencing identifies regulatory domain architecture at promoters and enhancers. Nat Genet. doi:10.1038/s41588-018-0234-5

Traut TW. 1994. Physiological concentrations of purines and pyrimidines. Mol Cell Biochem 140:1-22.

Tsai FTF, Sigler PB. 2000. Structural basis of preinitiation complex assembly on human Pol II promoters. EMBO J 19:25-36.

van de Geijn B, McVicker G, Gilad Y, Pritchard JK. 2015. WASP: allele-specific software for robust molecular quantitative trait locus discovery. Nat Methods 12:1061-1063.

Wang X, Soloway PD, Clark AG. 2011. A survey for novel imprinted genes in the mouse placenta by mRNA-seq. Genetics 189:109-122.

Wang Z, Chu T, Choate LA, Danko CG. 2018. Identification of regulatory elements from nascent transcription using dREG. Genome Res 29:293-303. 
bioRxiv preprint doi: https://doi.org/10.1101/2021.05.23.445279; this version posted May 30, 2021. The copyright holder has placed this preprint (which was not certified by peer review) in the Public Domain. It is no longer restricted by copyright. Anyone can legally share, reuse, remix, or adapt this material for any purpose without crediting the original authors.

Wilkins JF. 2014. Genomic imprinting of Grb10: coadaptation or conflict? PLoS Biol.

Zeitlinger J, Stark A, Kellis M, Hong J-W, Nechaev S, Adelman K, Levine M, Young RA. 2007. RNA polymerase stalling at developmental control genes in the Drosophila melanogaster embryo. Nat Genet 39:1512-1516. 